\title{
Flavor violating signatures of lighter and heavier Higgs bosons within the Two Higgs Doublet Model Type-III at the LHeC
}

\author{
S.P. Das \\ Instituto de Física, Benemérita Universidad Autónoma de Puebla, \\ Apdo. Postal J-48, C.P. 72570 Puebla, Puebla, \\ México and Facultad de Ciencias Físico-Matemáticas, Benemérita Universidad Autónoma de Puebla, \\ Apdo. Postal 1364, C.P. 72570 Puebla, Puebla, México.
}

\section{J. Hernández-Sánchez}

Facultad de Ciencias de la Electrónica, Benemérita Universidad Autónoma de Puebla, Apdo. Postal 542, C.P. 72570 Puebla, Puebla, México and Dual C-P Institute of High Energy Physics, México

\section{S. Moretti}

School of Physics and Astronomy, University of Southampton, Highfield, Southampton SO17 1BJ, United Kingdom, and Particle Physics Department, Rutherford Appleton Laboratory, Chilton, Didcot, Oxon OX11 OQX, United Kingdon用

A. Rosado,

Instituto de Física, Benemérita Universidad Autónoma de Puebla, Apdo. Postal J-48, C.P. 72570 Puebla, Puebla, México\$

\section{R. Xoxocotzi}

Facultad de Ciencias Físico-Matemáticas, Benemérita Universidad Autónoma de Puebla, Apdo. Postal 1364, C.P. 72570 Puebla, Puebla, México [0

(Dated: June 11, 2021)

We analyze the prospects for observing the light and heavy CP-even neutral Higgs bosons $(\phi=$ $h$ and $H$ ) in their decays into flavor violating $b \bar{s}$ channels (including charge conjugation) at the proposed Large Hadron electron Collider (LHeC), with $\sqrt{s} \approx 1.3 \mathrm{TeV}$, in the framework of a 2 Higgs Doublet Model (2HDM) Type-III, assuming a four-zero texture in the Yukawa matrices and a general Higgs potential. We consider theoretically consistent scenarios in agreement with current experimental data from flavor and Higgs physics. We investigate the charged current production process $\nu_{e} \phi q$ in presence of flavor violating decays of the Higgs bosons, that lead to a 3 -jets $+\mathbb{k}_{T}$ signature. We demand exactly two jets, one tagged $b$-jet and one light-flavor jet, all in the central rapidity region. The remaining jet (originated by the remnant quark $q$ ) is tagged in the forward or backward regions and this together with a central jet veto (not more than one light-flavor jet) are essential criterions to enhance the signal-to-background rates. We consider the most relevant Standard Model (SM) backgrounds, treating $c$-jets separately from light-flavor and gluon ones, while allowing for mis-tagging. We find that the SM-like Higgs boson, $h$, would be accessible within several parameter configurations of our model at approximately the $1-2 \sigma$ level with $100 \mathrm{fb}^{-1}$ of data. We also find that the heaviest neutral Higgs boson, $H$, with mass up to $150 \mathrm{GeV}$, would have a $1 \sigma$ significance for the same data sample. At the end of the LHeC running, one would have ten times data accumulation and for all the Higgs masses the significances are enhanced so as to allow for detection of both the $h$ and $H$ state. Hence, one of the most viable extensions of 2HDMs with Flavor Changing Neutral Currents (FCNCs) generated at tree level but controlled by a four-zero texture approach in the Yukawa matrices, as opposed to the adoption of ad hoc discrete symmetries, could be put under scrutiny at a future $e p$ machine.

\footnotetext{
*sprasad@ifuap.buap.mx

† jaime.hernandez@correo.buap.mx

$\ddagger$ s.moretti@soton.ac.uk

$\S$ rosado@ifuap.buap.mx

ฯ xoxo_reyna@yahoo.com.mx
} 


\section{INTRODUCTION}

The Standard Model (SM) is well established by now after the discovery of a Higgs boson by the ATLAS [1] and CMS 2] experiments at the Large Hadron Collider (LHC). However, when one considers some theoretical aspects of the SM, for example, lepton number violation, which is already manifested in the form of small but non-zero neutrino masses and actively searched for in the other two fermionic sectors (e.g., in $\mu \rightarrow e \gamma$ decays by the MEG experiment [3] and in B-physics by BaBar [4] and Belle [5] 1], one necessarily has to postulate New Physics (NP) Beyond the SM (BSM). By combining the evidence of Higgs states and the presence in Nature of flavor violation, then one is well motivated in searching for evidence of BSM physics in a context where the two aspects merge, i.e., in flavor violating Higgs boson decays. In general, limited to the Higgs sector, several BSM scenarios have been invoked by introducing extra Higgs singlets, doublets and/or triplets. As the Higgs boson discovery at the LHC is consistent with a doublet structure, we refrain here from considering BSM constructions with either of the other two aforementioned Higgs representations. Therefore, in staying with multi-Higgs doublet structures, the simplest of such Higgs scenarios is the so called 2-Higgs Doublet Model (2HDM) [6, 7], which will be the theoretical focus of our study.

Among the many phenomenological sides of a 2HDM, we are indeed concerned here with flavor violating Higgs boson decays in the quark sector, building on previous works of ours, see, e.g., Refs. [8 15]. However, plentiful of studies, also including lepton flavor violating scenarios, exist, some specific to $2 \mathrm{HDMs}$ and others adaptable to their case: see for an incomplete list, e.g., Refs. 16 32.

The actual search scope for Higgs bosons in flavor violating modes at the LHC has also been actively studied, see 33 40. It has also been investigated in the context of a future $e^{+} e^{-}[43$ ] and $\gamma \gamma$ collider [12]. Prospects at a future hadron collider have been investigated in [38].

Herein, we are particularly motivated by a possible enhancement of flavor violating quark decays $(\phi \rightarrow b \bar{s})$ of intermediate mass Higgs bosons (below the top-quark mass) and we will focus on the possibility to access such signatures at the possibly upcoming Large Hadron electron Collider (LHeC). The LHeC facility [44] presently discussed as possible at CERN in the near future would be a Deep Inelastic Scattering (DIS) experiment at the TeV scale, with center-of-mass energy of around $1.3 \mathrm{TeV}$. In comparison to the another recently closed (in 2007) DIS experiment (the Hadron-Electron Ring Accelerator (HERA) [45] at DESY of around $320 \mathrm{GeV}$ in energy with integrated luminosity of around $0.5 \mathrm{fb}^{-1}$ ), the LHeC might deliver data samples of approximately $100 \mathrm{fb}^{-1}$ and with a higher detector coverage. Further, the overall kinematic range (in $x$ and $Q^{2}$ ) accessible at LHeC is 20 times larger than at HERA.

While the primary task of a collider like the LHeC will be in-depth studies of QCD, the machine also affords some scope to study Higgs bosons decaying via flavor violating processes [46]. Our objective in this paper is to study the feasibility of finding two CP-even neutral Higgs bosons, the SM-like Higgs state $h$ and its heavier counterpart $H$, at the upcoming LHeC assuming as BSM framework a 2HDM Type-III (henceforth 2HDM-III for short), which embodies a four-zero texture approach in the Yukawa matrices as the mechanism to control Flavor Changing Neutral Currents (FCNCs). As we have shown in previous analyses, this is precisely the framework which establishes $\phi \rightarrow b \bar{s}+$ c.c. as an hallmark signature of quark flavor violation in the Higgs sector whose detectability is under investigation at the LHC and future $e^{+} e^{-}$and $\gamma \gamma$ collider.

The plan of our paper is as follows. In the next Section we describe briefly the theoretical structure of the 2HDM-III with a four-zero texture embedded in the Yukawa matrices. In Section III, we demarcate the allowed 2HDM-III parameter space in presence of both theoretical and experimental constraints. In Section IV, we explain the characteristics of the Higgs boson signal from charged current production. We introduce the most important SM backgrounds and finally, we carry out both a parameter space scan and a signal-to-backgrounds analysis for some characteristic benchmarks by adopting a simple cut-based optimization to isolate $\phi \rightarrow b \bar{s}+$ c.c. events. In section $\mathrm{V}$, we recap and present our conclusions.

\section{THE HIGGS-YUKAWA SECTOR OF THE 2HDM TYPE-III}

In the $2 \mathrm{HDM}$, the two Higgs scalar doublets, $\Phi_{1}^{\dagger}=\left(\phi_{1}^{-}, \phi_{1}^{0 *}\right)$ and $\Phi_{2}^{\dagger}=\left(\phi_{2}^{-}, \phi_{2}^{0 *}\right)$, have the same hypercharge +1 such that both couple to the same quark flavor. Since a specific four-zero texture is implemented as a flavor symmetry in the Yukawa sector, this is the mechanism which controls FCNCs so that discrete symmetries in the Higgs potential are not needed [8 12]. Then the most general $S U(2)_{L} \times U(1)_{Y}$ invariant scalar potential, following [7], can be written

\footnotetext{
1 In fact, also top-quark flavor violating decays into charm quarks and Higgs bosons are currently under investigation at the LHC.

2 Current experimental results at the LHC include both ATLAS [41] and CMS [42] analyses.
} 
as:

$$
\begin{aligned}
V\left(\Phi_{1}, \Phi_{2}\right)= & \mu_{1}^{2}\left(\Phi_{1}^{\dagger} \Phi_{1}\right)+\mu_{2}^{2}\left(\Phi_{2}^{\dagger} \Phi_{2}\right)-\left(\mu_{12}^{2}\left(\Phi_{1}^{\dagger} \Phi_{2}\right)+\text { H.c. }\right)+\frac{1}{2} \lambda_{1}\left(\Phi_{1}^{\dagger} \Phi_{1}\right)^{2} \\
& +\frac{1}{2} \lambda_{2}\left(\Phi_{2}^{\dagger} \Phi_{2}\right)^{2}+\lambda_{3}\left(\Phi_{1}^{\dagger} \Phi_{1}\right)\left(\Phi_{2}^{\dagger} \Phi_{2}\right)+\lambda_{4}\left(\Phi_{1}^{\dagger} \Phi_{2}\right)\left(\Phi_{2}^{\dagger} \Phi_{1}\right) \\
& +\left(\frac{1}{2} \lambda_{5}\left(\Phi_{1}^{\dagger} \Phi_{2}\right)^{2}+\left(\lambda_{6}\left(\Phi_{1}^{\dagger} \Phi_{1}\right)+\lambda_{7}\left(\Phi_{2}^{\dagger} \Phi_{2}\right)\right)\left(\Phi_{1}^{\dagger} \Phi_{2}\right)+\text { H.c. }\right),
\end{aligned}
$$

where all the parameters are assumed to be rea 3 , including the Vacuum Expectation Values (VEVs) of the scalar fields, hence there is no CP-violation. In general, by introducing some discrete symmetry $\Phi_{1} \rightarrow \Phi_{1}$ and $\Phi_{2} \rightarrow-\Phi_{2}$, the scalar potential does not have the contributions of $\lambda_{6}$ and $\lambda_{7}$.

It has long been proposed that there are four possibilities to satisfy the Paschos-Glashow-Weinberg theorem [47] in 2HDMs [6], [7]. These are defined as follows: Type I (where one Higgs doublet couples to all fermions); Type-II (where one Higgs doublet couples to up-type quarks and the other to down-type ones); Type-X (also called "Lepton-specific", where the quark couplings are Type-I and the lepton ones Type-II); Type Y (also called "Flipped" model, where the quark couplings are Type II and the lepton ones Type-I). With these two scalar doublets, there are eight fields but only five of them are physical (pseudo)scalar ("Higgs") fields, which correspond to: two neutral CP-even bosons $h$ (lighter) $H$ (heavier), one neutral CP-odd boson $A$ and two charged bosons $H^{ \pm}$. The mixing angle $\alpha$ of the two neutral $\mathrm{CP}$-even bosons $h$ and $H$ is another parameter of the 2HDM model. In total, the 2HDM model can be described by the parameters $\alpha, \beta$ (where $\tan \beta$ is the ratio of the VEVs of the two Higgs doublet fields) and the masses of the five Higgs particles. With these inputs one can estimate all the parameters that are present in the scalar potential, to be specific, the $\lambda$ 's. These $\lambda$ 's (together with various scalar mass parameters) enter the expressions of the theoretical constraints like: vacuum stability, unitarity, perturbativity and also various EW Precision Observables (EWPOs), for example, the oblique parameters. All these 2HDM types are fully compatible with the SM-like Higgs boson discovery.

The flavor sector of 2HDMs is testable in low as well as in high energy collider experiments. The tests have been carried out in the most general version of a 2HDM with a Yukawa four-zero texture, wherein the Yukawa couplings are proportional to the geometric mean of two fermions masses, $g_{i j} \propto \sqrt{\left(m_{i} m_{j}\right)} \chi_{i j}$ [48, 49]. As it was mentioned, a consequence of this is that the terms of the scalar potential including $\lambda_{6}$ and $\lambda_{7}$ should now be taken into account. This leads to tri-linear and quartic self-couplings of the scalar fields [8, 12] affecting the model phenomenology in one loop processes via di-Higgs and tri-Higgs topologies, both in production and decay processes. It has been shown that the EWPO $\rho$ can deviate from experimental bounds at one loop level, as long as the mass difference between charged Higgs bosons with CP-even/CP-odd masses is large, irrespective of the value of $\lambda_{6}$ and $\lambda_{7}$. Hence, some level of degeneracy between one neutral and the charged Higgs states is a precondition on the 2HDM spectra . In our construction, the Yukawa Lagrangian [8] is given by:

$$
\mathcal{L}_{Y}=-\left(Y_{1}^{u} \bar{Q}_{L} \tilde{\Phi}_{1} u_{R}+Y_{2}^{u} \bar{Q}_{L} \tilde{\Phi}_{2} u_{R}+Y_{1}^{d} \bar{Q}_{L} \Phi_{1} d_{R}+Y_{2}^{d} \bar{Q}_{L} \Phi_{2} d_{R}+Y_{1}^{l} \overline{L_{L}} \Phi_{1} l_{R}+Y_{2}^{l} \overline{L_{L}} \Phi_{2} l_{R}\right),
$$

where $\Phi_{1,2}=\left(\phi_{1,2}^{+}, \phi_{1,2}^{0}\right)^{T}$ refer to the two Higgs doublets, $\tilde{\Phi}_{1,2}=i \sigma_{2} \Phi_{1,2}^{*}$. Besides, the fermion mass matrices after EW symmetry breaking are given, from Eq. (2), by: $M_{f}=\frac{1}{\sqrt{2}}\left(v_{1} Y_{1}^{f}+v_{2} Y_{2}^{f}\right), f=u$, $d$, $l$, assuming that both Yukawa matrices $Y_{1}^{f}$ and $Y_{2}^{f}$ have the four-zero-texture form and are Hermitian [10, 14, 48]. After diagonalisation, $\bar{M}_{f}=V_{f L}^{\dagger} M_{f} V_{f R}$, one has $\bar{M}_{f}=\frac{1}{\sqrt{2}}\left(v_{1} \tilde{Y}_{1}^{f}+v_{2} \tilde{Y}_{2}^{f}\right)$, where $\tilde{Y}_{i}^{f}=V_{f L}^{\dagger} Y_{i}^{f} V_{f R}$. One can obtain a compact and generic form for the rotated matrix $\tilde{Y}_{n}^{f} 4$ :

$$
\left[\tilde{Y}_{n}^{f}\right]_{i j}=\frac{\sqrt{m_{i}^{f} m_{j}^{f}}}{v}\left[\tilde{\chi}_{n}^{f}\right]_{i j}=\frac{\sqrt{m_{i}^{f} m_{j}^{f}}}{v}\left[\chi_{n}^{f}\right]_{i j} e^{i \vartheta_{i j}^{f}}
$$

where the $\chi$ 's are unknown dimensionless parameters of the model. Following [10], one has a generic expression for the couplings of the Higgs bosons to the fermions given as

$$
\begin{aligned}
\mathcal{L}^{\bar{f}_{i} f_{j} \phi}= & -\left\{\frac{\sqrt{2}}{v} \bar{u}_{i}\left(m_{d_{j}} X_{i j} P_{R}+m_{u_{i}} Y_{i j} P_{L}\right) d_{j} H^{+}+\frac{\sqrt{2} m_{l_{j}}}{v} Z_{i j} \overline{\nu_{L}} l_{R} H^{+}+H . c .\right\} \\
& -\frac{1}{v}\left\{\bar{f}_{i} m_{f_{i}} h_{i j}^{f} f_{j} h^{0}+\bar{f}_{i} m_{f_{i}} H_{i j}^{f} f_{j} H^{0}-i \bar{f}_{i} m_{f_{i}} A_{i j}^{f} f_{j} \gamma_{5} A^{0}\right\}
\end{aligned}
$$

\footnotetext{
3 The $\mu_{12}^{2}, \lambda_{5}, \lambda_{6}$ and $\lambda_{7}$ parameters could be complex in general, but for simplicity we assume these parameters to be real.

4 We have shown in several parametrisations that this structure corresponds, as a particular case, to the Cheng and Sher ansatz 9 , 10 , 15, 48.
} 
where $\phi_{i j}^{f}(\phi=h, H, A), X_{i j}, Y_{i j}$ and $Z_{i j}$ are defined as follows:

$$
\begin{aligned}
& h_{i j}^{d}=\xi_{h}^{d} \delta_{i j}+\frac{\left(\xi_{H}^{d}-X \xi_{h}^{d}\right)}{\sqrt{2} f(X)} \sqrt{\frac{m_{d_{j}}}{m_{d_{i}}}} \tilde{\chi}_{i j}^{d}, \quad h_{i j}^{\ell}=h_{i j}^{d}(d \rightarrow \ell, X \rightarrow Z), \\
& H_{i j}^{d}=\xi_{H}^{d} \delta_{i j}-\frac{\left(\xi_{h}^{d}+X \xi_{H}^{d}\right)}{\sqrt{2} f(X)} \sqrt{\frac{m_{d_{j}}}{m_{d_{i}}}} \tilde{\chi}_{i j}^{d}, \quad H_{i j}^{\ell}=H_{i j}^{d}(d \rightarrow \ell, X \rightarrow Z), \\
& A_{i j}^{d}=-X \delta_{i j}+\frac{f(X)}{\sqrt{2}} \sqrt{\frac{m_{d_{j}}}{m_{d_{i}}}} \tilde{\chi}_{i j}^{d}, \quad A_{i j}^{\ell}=A_{i j}^{d}(d \rightarrow \ell, X \rightarrow Z), A_{i j}^{u}=A_{i j}^{d}(d \rightarrow u, X \rightarrow Y), \\
& h_{i j}^{u}=\xi_{h}^{u} \delta_{i j}-\frac{\left(\xi_{H}^{u}+Y \xi_{h}^{u}\right)}{\sqrt{2} f(Y)} \sqrt{\frac{m_{u_{j}}}{m_{u_{i}}}} \tilde{\chi}_{i j}^{u}, \quad H_{i j}^{u}=\xi_{H}^{u} \delta_{i j}+\frac{\left(\xi_{h}^{u}-Y \xi_{H}^{u}\right)}{\sqrt{2} f(Y)} \sqrt{\frac{m_{u_{j}}}{m_{u_{i}}}} \tilde{\chi}_{i j}^{u}, \\
& X_{i j}=\sum_{l=1}^{3}\left(V_{\mathrm{CKM}}\right)_{i l}\left[X \frac{m_{d_{l}}}{m_{d_{j}}} \delta_{l j}-\frac{f(X)}{\sqrt{2}} \sqrt{\frac{m_{d_{l}}}{m_{d_{j}}}} \tilde{\chi}_{l j}^{d}\right] \\
& Y_{i j}=\sum_{l=1}^{3}\left[Y \delta_{i l}-\frac{f(Y)}{\sqrt{2}} \sqrt{\frac{m_{u_{l}}}{m_{u_{i}}}} \tilde{\chi}_{i l}^{u}\right]\left(V_{\mathrm{CKM})_{l j}},\right. \\
& \left.Z_{i j} \frac{m_{l_{i}}}{m_{l_{j}}} \delta_{i j}-\frac{f(Z)}{\sqrt{2}} \sqrt{\frac{m_{l_{i}}}{m_{l_{j}}}} \tilde{\chi}_{i j}^{l}\right]
\end{aligned}
$$

where $f(x)=\sqrt{1+x^{2}}, \xi_{\phi}^{f}$ are related to the trigonometric ratios (i.e., $\cos \alpha / \sin \beta, \sin \alpha / \sin \beta, \cos \alpha / \cos \beta$, $\sin \alpha / \cos \beta$ ) and the parameters $X, Y$ and $Z$ can be related to $\tan \beta$ or $\cot \beta$, according to the various incarnations of $2 \mathrm{HDMs}$ [10] (see the Table [1). Taking into account that the Higgs-fermion-fermion $(\phi f f)$ coupling in the $2 \mathrm{HDM}-\mathrm{III}$ is written as $g_{2 \mathrm{HDM}-\mathrm{III}}^{\phi f f}=g_{2 \mathrm{HDM}-\text { any }}^{\phi f f}+\Delta g$, where $g_{2 \mathrm{HDM} \text {-any }}^{\phi f f}$ is the coupling $\phi f f$ in some of the $2 \mathrm{HDM}$ with discrete symmetry and $\Delta g$ is the contribution of the four-zero texture5, it was pointed out in [10] that this Lagrangian could also represent a Multi-Higgs Doublet Model (MHDM) or an Aligned 2HDM (A2HDM) with additional flavor physics in the Yukawa matrices.

\begin{tabular}{|c|c|c|c|c|c|c|c|c|c|}
\hline \hline 2HDM-III & $X$ & $Y$ & $Z$ & $\xi_{h}^{u}$ & $\xi_{h}^{d}$ & $\xi_{h}^{\ell}$ & $\xi_{H}^{u}$ & $\xi_{H}^{d}$ & $\xi_{H}^{\ell}$ \\
\hline \hline 2HDM-I-Like & $-c t_{\beta}$ & $c t_{\beta}$ & $-c t_{\beta}$ & $c_{\alpha} / s_{\beta}$ & $c_{\alpha} / s_{\beta}$ & $c_{\alpha} / s_{\beta}$ & $s_{\alpha} / s_{\beta}$ & $s_{\alpha} / s_{\beta}$ & $s_{\alpha} / s_{\beta}$ \\
\hline 2HDM-II-Like & $t_{\beta}$ & $c t_{\beta}$ & $t_{\beta}$ & $c_{\alpha} / s_{\beta}$ & $-s_{\alpha} / c_{\beta}$ & $-s_{\alpha} / c_{\beta}$ & $s_{\alpha} / s_{\beta}$ & $c_{\alpha} / c_{\beta}$ & $c_{\alpha} / c_{\beta}$ \\
\hline 2HDM-X-Like & $-c t_{\beta}$ & $c t_{\beta}$ & $t_{\beta}$ & $c_{\alpha} / s_{\beta}$ & $c_{\alpha} / s_{\beta}$ & $-s_{\alpha} / c_{\beta}$ & $s_{\alpha} / s_{\beta}$ & $s_{\alpha} / s_{\beta}$ & $c_{\alpha} / c_{\beta}$ \\
\hline 2HDM-Y-Like & $t_{\beta}$ & $c t_{\beta}$ & $-c t_{\beta}$ & $c_{\alpha} / s_{\beta}$ & $-s_{\alpha} / c_{\beta}$ & $c_{\alpha} / s_{\beta}$ & $s_{\alpha} / s_{\beta}$ & $c_{\alpha} / c_{\beta}$ & $s_{\alpha} / s_{\beta}$ \\
\hline
\end{tabular}

TABLE I. Parameters $\xi_{\phi}^{f}(\phi=h, H, A$ with $f=u, d, \ell), X, Y$ and $Z$ as defined in Eqs. (5)-(11) for four versions of the 2HDM-III. These parameters are related to the interactions of Higgs bosons with the fermions given in Eq. (44). Here, $t_{\beta}=\tan \beta, c t_{\beta}=\cot \beta, s_{\alpha}=\sin \alpha, c_{\alpha}=\cos \alpha, s_{\beta}=\sin \beta, c_{\beta}=\cos \beta$.

Here, we consider three different incarnations of the 2HDM-III, which correspond to to the four 2HDM types already described except the lepton specific one, as here leptonic Branching Ratios (BRs) are dominant, whereas we intend to look for an enhancement in the Higgs to $b \bar{s}$ decay because of flavor violation. We will finally show that, in different scenarios of the 2HDM-III, a substantial enhancement of the $\mathrm{BR}(\phi \rightarrow b \bar{s})$ (including charge conjugation) is possible. We do so first via a parameter scan of the 2HDM-III at the inclusive level, followed by the detailed event generation analysis of some benchmark scenarios amenable to phenomenological investigation.

\section{THE 2HDM-III PARAMETERS AND BENCHMARKS}

In this section, we will perform a parameter scan of the 2HDM-III of interest from which we will extract our benchmark scenarios, all of which will be studied in our final signal-to-background simulations, albeit we will show detailed results only for a subset of these for reasons of space.

First, we ought to explain the constraints we have enforced in our analysis. As for the experimental ones, we have taken into account recent experimental bounds from flavor physics [9, 10]: i.e., from $B \rightarrow \tau \nu_{\tau}, D \rightarrow \mu \nu, D s \rightarrow \ell \nu$,

\footnotetext{
${ }^{5}$ For example, one can recovers the Yukawa interactions given in Refs. [50 52$]$ with $\chi_{i j}^{f}=0$.
} 
the semileptonic transition $B \rightarrow D \tau \nu_{\tau}$, the inclusive decay $B \rightarrow X_{s} \gamma, B_{0}-B_{0}$ mixing, $B_{s} \rightarrow \mu^{+} \mu^{-}$and the radiative decay $Z \rightarrow b \bar{b}$. (We have also imposed EWPO limits.) On the theoretical side, we have enforced perturbativity, triviality, vacuum stability and unitarity constraints [8, 53. In all the constraints mentioned above, the charged Higgs bosons masses are the crucial parameter, as diagrams with $H^{ \pm}$states co-exist alongside those involving the SM $W^{ \pm}$ exchange diagrams. In this connection, alongside flavor and EWPO constraints, we have also accounted for those stemming from Tevatron and LHC searches [10, 11, 54 56. 6 .

\section{A. Parameter scan}

We scan the parameters space of the model and we only consider as viable the points that avoid the aforementioned theoretical and experimental bounds and that are fully consistent with the most recent results of Higgs physics from LHC. Taking into account that our model provides interesting new physics in the form of a substantial enhancement of the decay $\phi \rightarrow s \bar{b}+h . c$. $(\phi=h, H)$, as a direct consequence of the off-diagonal terms of the texture of the Yukawa matrices. In our scanning, we ask that $\operatorname{BR}(\phi \rightarrow s \bar{b}+$ h.c. $) \sim 0.01$ to 0.1 , keeping the decay $\phi \rightarrow b \bar{b}$ dominant. Firstly, we scan the off-diagonal terms of the Yukawa matrices and after we chose some interesting sets of the $\chi$ 's parameters, which are consistent with the flavor physics constraints and Higgs physics bounds used in the analysis of [9 11], where we have shown that several meson-physics processes are very sensitive to charged Higgs boson exchange, and the off-diagonal terms of Yukawa matrices given in the Eq. (3) are kept constrained in the following range:

$$
-0.06 \leq\left(\chi_{n}^{d}\right)_{23} \leq 0.3, \quad-0.3 \leq\left(\chi_{n}^{u}\right)_{23} \leq 0.5 .
$$

Secondly, we fix the $\chi$ 's parameters and the masses of the following Higgs bosons, $m_{h}=125 \mathrm{GeV}, m_{A}=100 \mathrm{GeV}$ and $m_{H^{ \pm}}=110 \mathrm{GeV}$. We run the mass $m_{H}$ of the Higgs boson $H$ from $130 \mathrm{GeV}$ up to $200 \mathrm{GeV}$. Therefore, we can reduce the study of the parameter space to that of the couplings $X$ and $Y$ only, which are constrained strongly by the inclusive radiative decay $B \rightarrow X_{s} \gamma$ through the following bound:

$$
-1.7 \leq \operatorname{Re}\left(\frac{X_{33} Y_{32}}{V_{t b} V_{V t s}}\right) \leq 0.3
$$

where $X_{33}, Y_{32}$ are defined in Eqs. (9)-(10) and $V_{t b}$ and $V_{t s}$ are elements of the Cabibbo-Kobayashi-Maskawa (CKM) matrix. From the constraint in Eq. (13), we can define the allowed region for two general cases. (a) For the case I defined by: $X=-Y$ or $X=Y, 0.1 \leq \cos (\beta-\alpha) \leq 0.5$, and fixing the parameters of Yukawa matrices, $\chi_{k k}^{u}=1.5$ $(k=2,3), \chi_{22}^{d}=1.8, \chi_{33}^{d}=1.2, \chi_{23}^{u, d}=0.2, \chi_{22}^{\ell}=0.5, \chi_{33}^{\ell}=1.2, \chi_{23}^{\ell}=0.1$. One can see in Fig. 1 in the left panel the allowed region for $Y=-X \leq 15$ and for the case $X=Y \leq 20$. This region could represent the case of the 2HDM-I plus a deviation given for the flavor symmetry of the Yuwaka matrices. (b) For the case II given by: $X>>Y$, with $\cos (\beta-\alpha)=0.1, \chi_{22}^{u}=0.5, \chi_{33}^{u}=1.4, \chi_{22}^{d}=2, \chi_{33}^{d}=1.3, \chi_{23}^{u}=-0.53, \chi_{23}^{d}=0.2, \chi_{22}^{\ell}=0.4, \chi_{33}^{\ell}=1.2$, we can see that the large values for parameter $\mathrm{X}$ is permitted. This case could be the incarnation of both the $2 \mathrm{HDM}-\mathrm{II}$ and 2HDM-Y (or flipped model) plus a deviation given by the four-zero texture of the Yukawa matrices. Considering these criteria, we chose three interesting scenarios from the versions of 2HDM-III given in the Tab. I] Scenario Ib which is related to the 2 HDM-I-Like, with $\cos (\beta)=0.5$, Scenario IIa is the case 2 HDM-II-like, with $\cos (\beta)=0.1$, and Scenario Ya is the case $2 \mathrm{HDM}-\mathrm{Y}$-like, with $\cos (\beta)=0.1$.

\section{B. Benchmark scenarios}

Taking in account the scan of the parameters space, we chose the benchmark scenarios, where their main features can be recapped as follows:

- Scenario Ib: 2HDM-III as 2HDM-I, with the couplings $\phi f f$ given by $g_{2 \mathrm{HDM}-\mathrm{III}}^{\phi f f}=g_{2 \mathrm{HDM}-\mathrm{I}}^{\phi f f}+\Delta g$ and $\cos (\beta-$ $\alpha)=0.5, \chi_{k k}^{u}=1.5(k=2,3), \chi_{22}^{d}=1.8, \chi_{33}^{d}=1.2, \chi_{23}^{u, d}=0.2, \chi_{22}^{\ell}=0.5, \chi_{33}^{\ell}=1.2, \chi_{23}^{\ell}=0.1, m_{A}=100 \mathrm{GeV}$ and $m_{H^{ \pm}}=110 \mathrm{GeV}$, taking $X$ and $Y$ located in the blue region of the left panel from Figure 1

- Scenario IIa: 2HDM-III as 2HDM-II, namely, the couplings $\phi f f$ given by $g_{2 \mathrm{HDM}-\mathrm{III}}^{\phi f f}=g_{2 \mathrm{HDM}-\mathrm{II}}^{\phi f f}+\Delta g$ and $\cos (\beta-\alpha)=0.1, \chi_{22}^{u}=0.5, \chi_{33}^{u}=1.4, \chi_{22}^{d}=2, \chi_{33}^{d}=1.3, \chi_{23}^{u}=-0.53, \chi_{23}^{d}=0.2, \chi_{22}^{\ell}=0.4, \chi_{33}^{\ell}=1.2$, $\chi_{23}^{\ell}=0.1, m_{A}=100 \mathrm{GeV}$ and $m_{H^{ \pm}}=110 \mathrm{GeV}$, taking $X$ and $Y$ allowed in the right panel of the Figure 1 .

${ }^{6}$ Current low energy constraints on the Higgs boson masses have been studied very recently [57, 58]. 

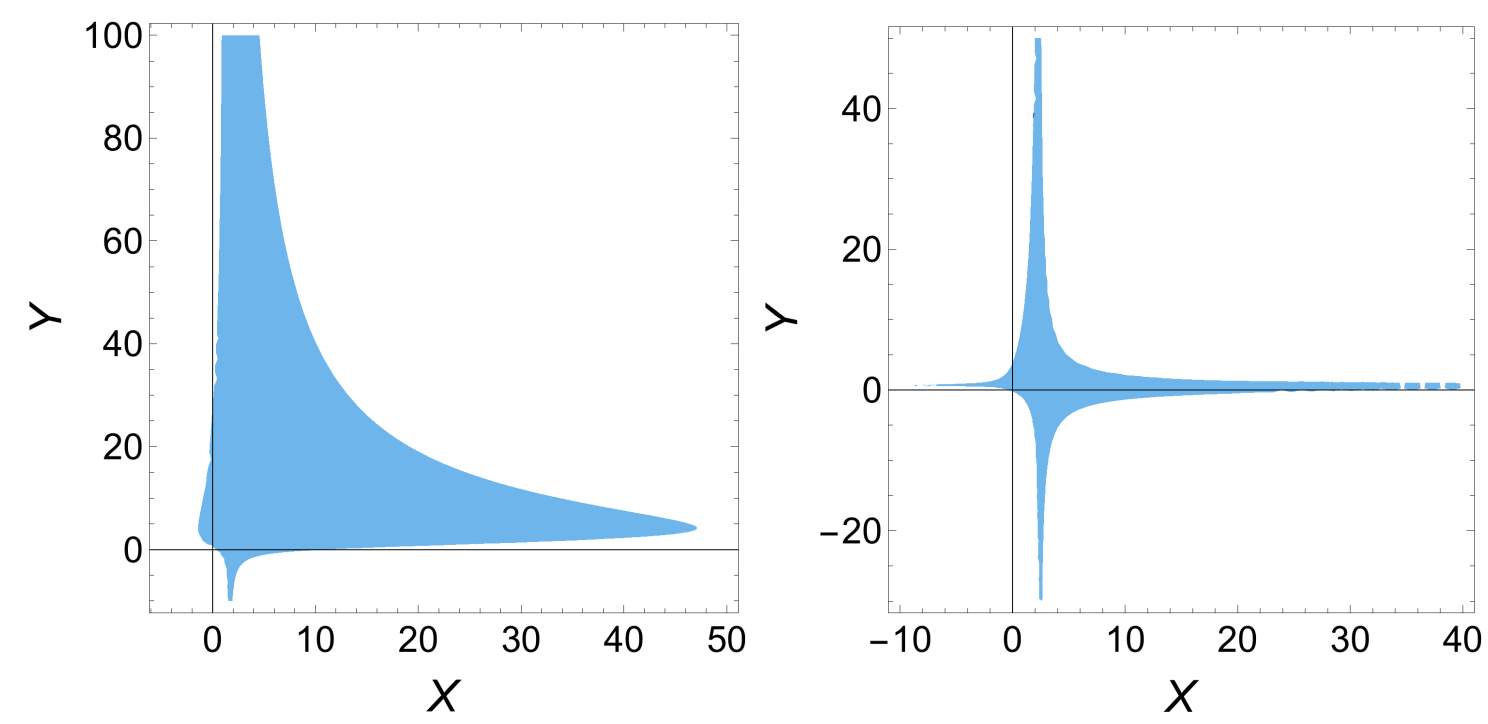

FIG. 1. The allowed region in the plane $X$ vs $Y$, using the constraint Eq. (13), which is obtained from the radiative inclusive decay $B \rightarrow X_{s} \gamma$. We obtain the Scenario Ib, which is shown in the left panel, with $0.1 \leq \cos (\beta-\alpha) \leq 0.5, \chi_{k k}^{u}=1.5(k=2,3)$, $\chi_{22}^{d}=1.8, \chi_{33}^{d}=1.2, \chi_{23}^{u, d}=0.2, \chi_{22}^{\ell}=0.5, \chi_{33}^{\ell}=1.2, \chi_{23}^{\ell}=0.1$. For Scenario IIa and Y, the allowed region is given in the right panel with $\cos (\beta-\alpha)=0.1, \chi_{22}^{u}=0.5, \chi_{33}^{u}=1.4, \chi_{22}^{d}=2, \chi_{33}^{d}=1.3, \chi_{23}^{u}=-0.53, \chi_{23}^{d}=0.2, \chi_{22}^{\ell}=0.4, \chi_{33}^{\ell}=1.2$, $\chi_{23}^{\ell}=0.1$. For both cases $m_{h}=125 \mathrm{GeV}, 130 \mathrm{GeV} \leq m_{H} \leq 300 \mathrm{GeV}, 100 \mathrm{GeV} \leq m_{A} \leq 250 \mathrm{GeV}, 110 \mathrm{GeV} \leq m_{H} \leq 200$ $\mathrm{GeV}$.

- Scenario Y: 2HDM-III as 2HDM-Y, namely, the couplings $\phi f f$ given by $g_{2 \mathrm{HDM}-\mathrm{III}}^{\phi f f}=g_{2 \mathrm{HDM}-\mathrm{Y}}^{\phi f f}+\Delta g$ and $\cos (\beta-\alpha)=0.1, \chi_{22}^{u}=0.5, \chi_{33}^{u}=1.4, \chi_{22}^{d}=2, \chi_{33}^{d}=1.3, \chi_{23}^{u}=-0.53, \chi_{23}^{d}=0.2, \chi_{22}^{\ell}=0.4, \chi_{33}^{\ell}=1.1$, $\chi_{23}^{\ell}=0.1, m_{A}=100 \mathrm{GeV}$ and $m_{H^{ \pm}}=110 \mathrm{GeV}$, taking the same $X$ and $Y$ for the Scenario IIa.

Hereinafter, we only simulated benchmarks where $\sigma \cdot B R(\phi \rightarrow b \bar{s})$ (cross section of the charged current production $\nu_{e} \phi q$ multiplied by Branching Ratio of the channel decay $\phi \rightarrow b \bar{s}+$ c.c., with $\left.(\phi=h, H)\right)$, are more than 0.15 fb so that, for an integrated luminosity of $100 \mathrm{fb}^{-1}$, we can start with at least 15 events. Finally, when producing differential spectra of physical observables, we will concentrate on three 2HDM scenarios where the number of Higgs signal events in the $b \bar{s}+$ c.c. mode are large enough in order to be able to appreciate the underlying dynamics.

\section{NUMERICAL ANALYSIS}

In this section, we describe first the production of Higgs signal. We then discuss the most important SM backgrounds and the different kinematics selections on the simulated events.

\section{A. Higgs bosons signals}

We consider the leading production processe 7 of Higgs boson: $\nu_{e} \phi q$, where $\phi=h$ and $H$ while $q$ is a light-flavor quark (i.e., $u, d, s, c)$. We assume that $\phi$ is dominantly decaying into $b \bar{s}$ (plus charge conjugation). So both of our signals, the lighter Higgs as well as the heavier Higgs one, contain three jet (one is forward and two are central), missing (transverse) energy and no-lepton. Out of the two central jets, one is $b$-tagged and the other is a light-flavor jet. We estimated the parton level signal cross sections with flavor-violation within the $2 \mathrm{HDM}-\mathrm{III}$ by using CalcHEP 59]. This implementation also calculates the BRs of the Higgs boson $\phi$ into $b \bar{s}$. For estimating the cross sections at the LHeC [44, 60 64], we consider an electron beam, of energy $E_{e^{-}}=60 \mathrm{GeV}$ and a proton beam of energy $E_{p}=7000$ $\mathrm{GeV}$, corresponding to a center-of-mass energy of approximately $\sqrt{s}=1.296 \mathrm{TeV}$. The integrated luminosity is 100 $\mathrm{fb}^{-1}$. To estimate the event rates at parton level we applied the following basic pre-selections:

$$
p_{T}^{q}>15 \mathrm{GeV}, \quad \Delta R(q, q)>0.4
$$

\footnotetext{
7 The charged-current production is approximately 5 times larger than the neutral current production. Moreover, the neutral current
} production contains an electron and, since we are vetoing leptons in this particular analysis, we consider only the charged processes. 
with $\Delta R=\Delta \eta^{2}+\Delta \phi^{2}$, where $\eta$ and $\phi$ are the pseudo-rapidity and azimuthal angle, respectively. We take $m_{t}=173.3$ $\mathrm{GeV}$ as the top-quark pole mass. We set the renormalization and factorization scale at the $Z$-boson mass (which is approximately the momentum transfer scale for the signal) and adopt CTEQ6L [65] as Parton Distribution Functions (PDFs), with $\alpha_{\mathrm{s}}$ (the strong coupling constant) evaluated consistently at all stages (PDFs, hard scattering and decays).

Considering the latter, we calculate in the allowed regions given above in the Figure 1, the event rates $(\sigma . B R . L)$ at parton level for the neutral Higgs bosons $h$ and $H$ in the Scenarios Ib, IIa and Y, respectively, considering both luminosities of $100 \mathrm{fb}^{-1}$ (left panel) and $1000 \mathrm{fb}^{-1}$ (right panel), which are shown in the Figures 20 One can see that the blue region contains the best benchmark points for all scenarios. We show that the most optimistic is in fact Scenario Ib for both Higgs bosons $h$ and $H$, which reach events rates of order 500-1300 (5000-13000) with an integrated luminosity of $100 \mathrm{fb}^{-1}\left(1000 \mathrm{fb}^{-1}\right)$, although Scenarios IIa and Y also have some interesting benchmark points where one can obtain 17 events rates at the same luminosity. The Tab. П shows the benchmark points that we select as interesting for studies at the LHeC. There are twenty-seven in total, obtained by taking the same three different values of the $H$ mass $\left(m_{H}=130,150,170 \mathrm{GeV}\right)$ in correspondence to nine different configurations of the other parameters. The product of cross sections times the relevant BRs $(\sigma . b s)$ are shown in Tab. I]
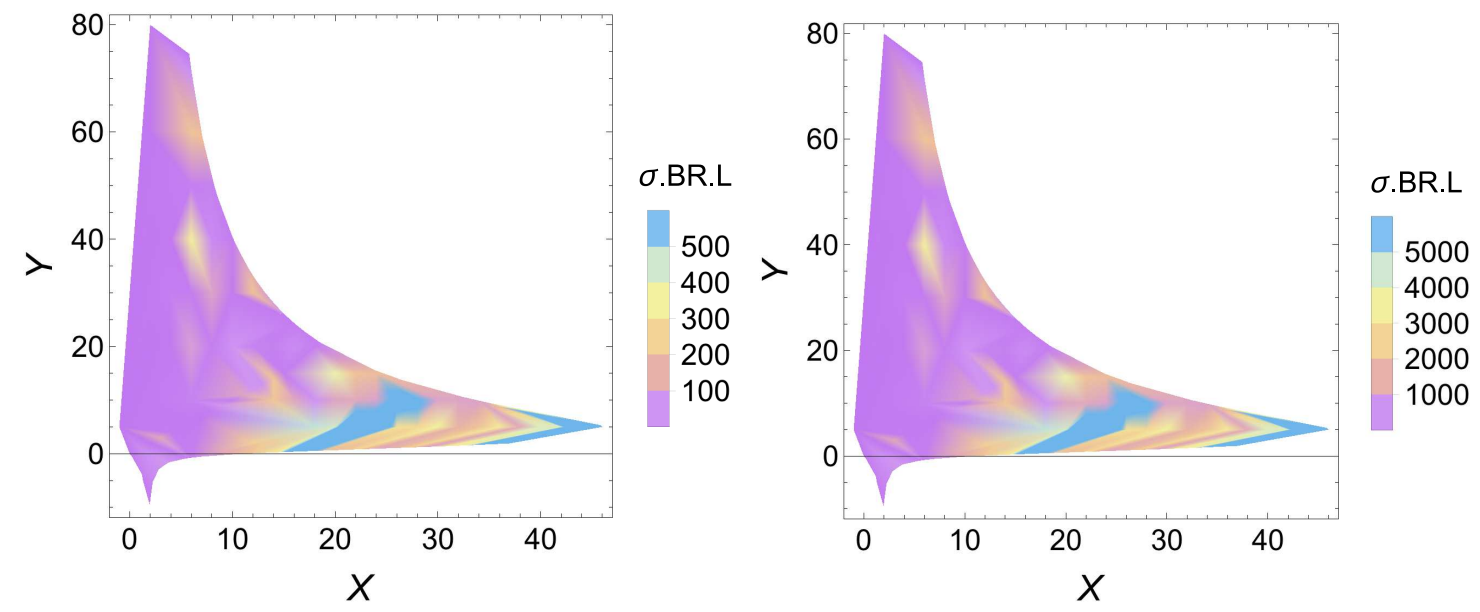

FIG. 2. Event rates ( $\sigma . B R . L)$ at parton level for the neutral Higgs boson $h$, where $L$ is the integrated luminosity. We show Scenario Ib for $100 \mathrm{fb}^{-1}$ (left panel) and $1000 \mathrm{fb}^{-1}$ (right panel). We consider $m_{h}=125 \mathrm{GeV}$.
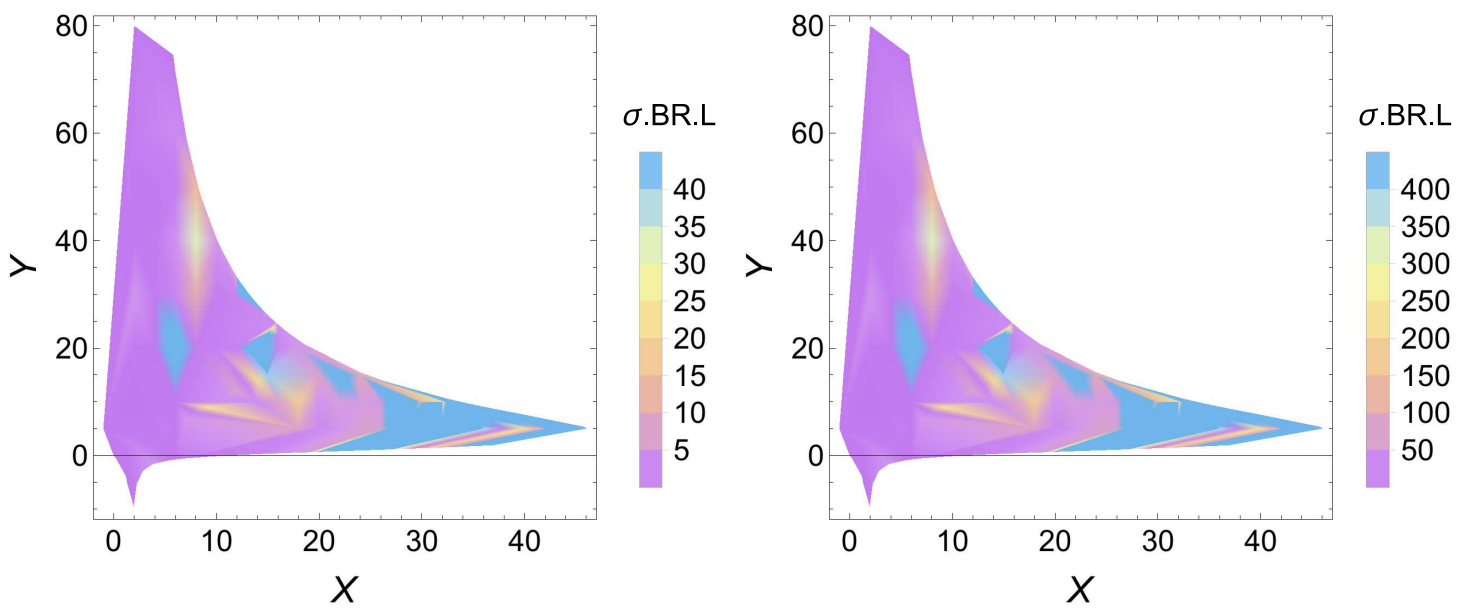

FIG. 3. Event rates ( $\sigma . B R . L)$ at parton level for the neutral Higgs boson $H$, where $L$ is the integrated luminosity. We show Scenario Ib for $100 \mathrm{fb}^{-1}$ (left panel) and $1000 \mathrm{fb}^{-1}$ (right panel). We consider $m_{H}=130$.

Restricting ourselves to the points for which the inclusive event rates are most optimistic, all estimated by taking all the light-flavor quarks, the $b$-quark and the gluon as fluxes inside the proton and upon considering appropriate flavor-mixing where appropriate, we have then proceeded as follows. The top-quark and $W$-boson were allowed to decay freely as implemented in PYTHIA [66]. Following this, it was recognized that the signal processes have unique 

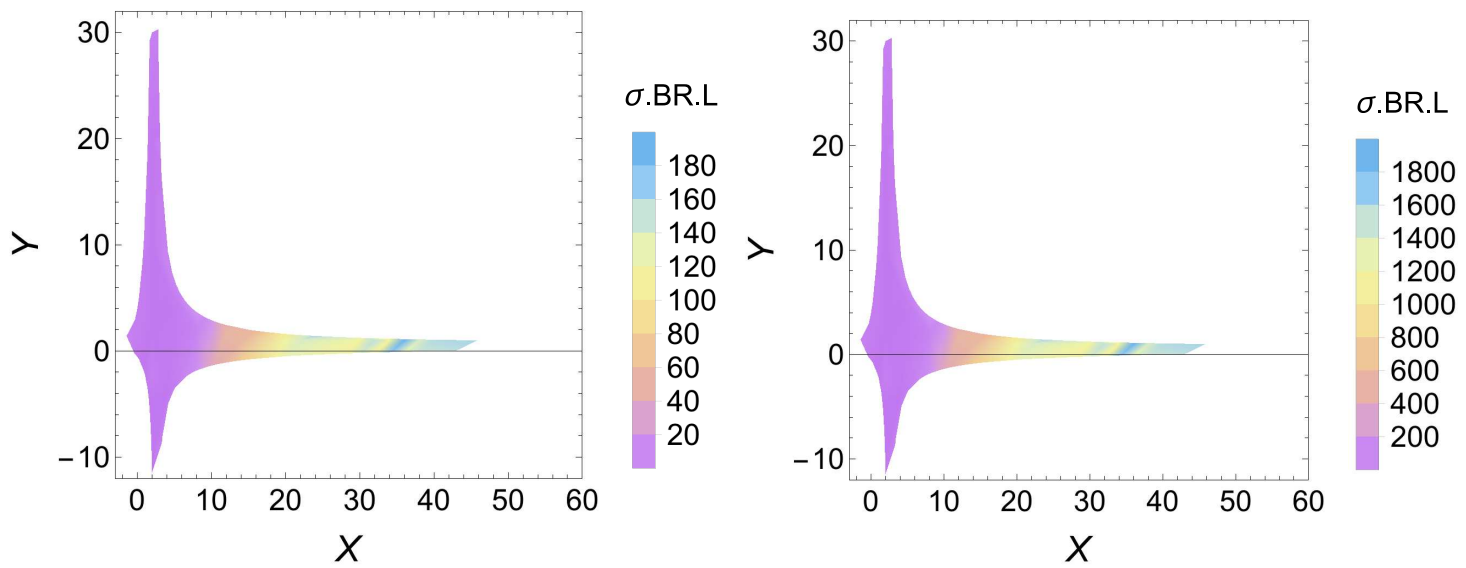

FIG. 4. Event rates $(\sigma . B R . L)$ at parton level for the neutral Higgs boson $h$, where $L$ is the integrated luminosity. We show Scenario IIa for $100 \mathrm{fb}^{-1}$ (left panel) and $1000 \mathrm{fb}^{-1}$ (right panel). We consider $m_{h}=125 \mathrm{GeV}$.
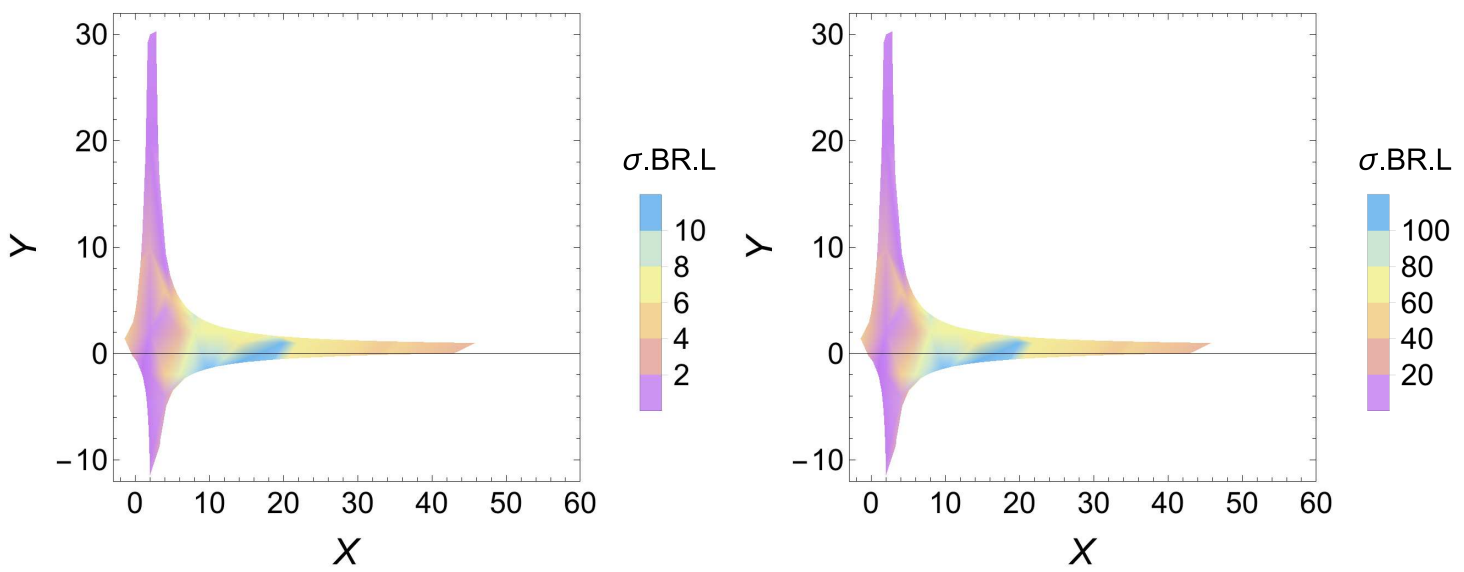

FIG. 5. Event rates ( $\sigma . B R . L)$ at parton level for the neutral Higgs boson $H$, where $L$ is the integrated luminosity. We show Scenario IIa for $100 \mathrm{fb}^{-1}$ (left panel) and $1000 \mathrm{fb}^{-1}$ (right panel). We consider $m_{H}=130$.
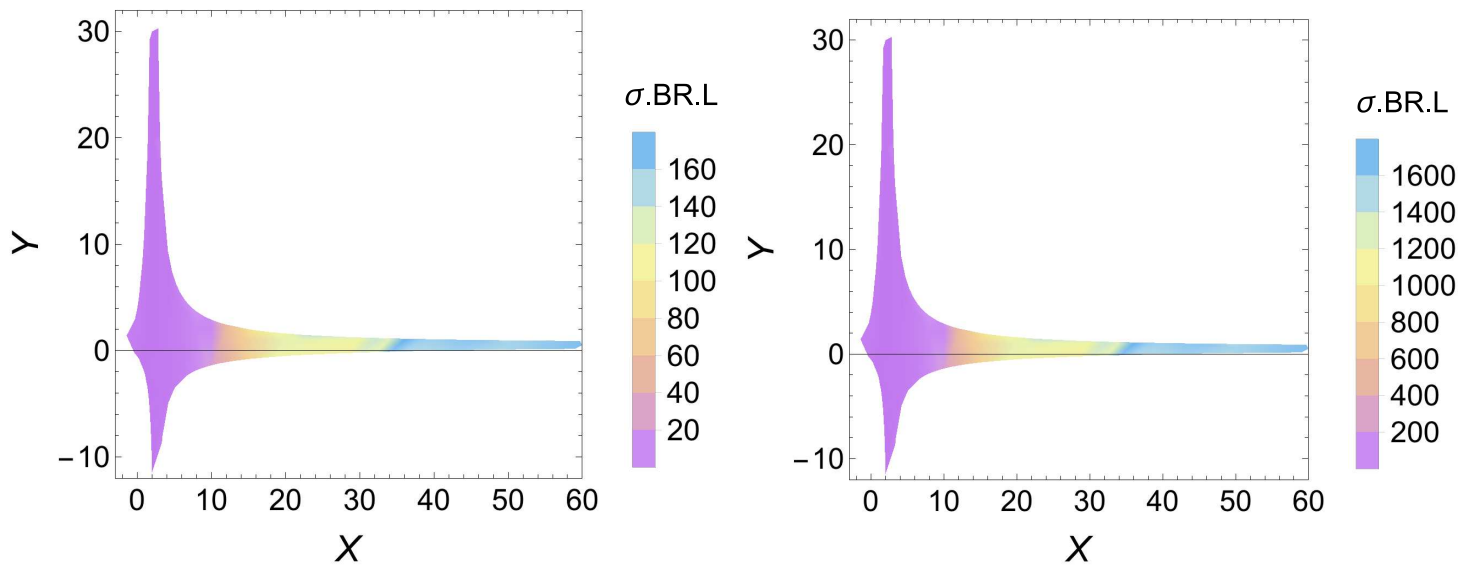

FIG. 6. Event rates $(\sigma . B R . L)$ at parton level for the neutral Higgs boson $h$, where $L$ is the integrated luminosity. We show Scenario Y for $100 \mathrm{fb}^{-1}$ (left panel) and $1000 \mathrm{fb}^{-1}$ (right panel). We consider $m_{h}=125 \mathrm{GeV}$.

kinematic profiles. In particular, the final state quark transverse momentum is less than the mass of the vector bosons, its energy is very high with a small angle with respect to the beam directions (i.e., high forward rapidity). This will serve as guidance in our event selection. However, before proceeding further in this direction, we have to acknowledge 

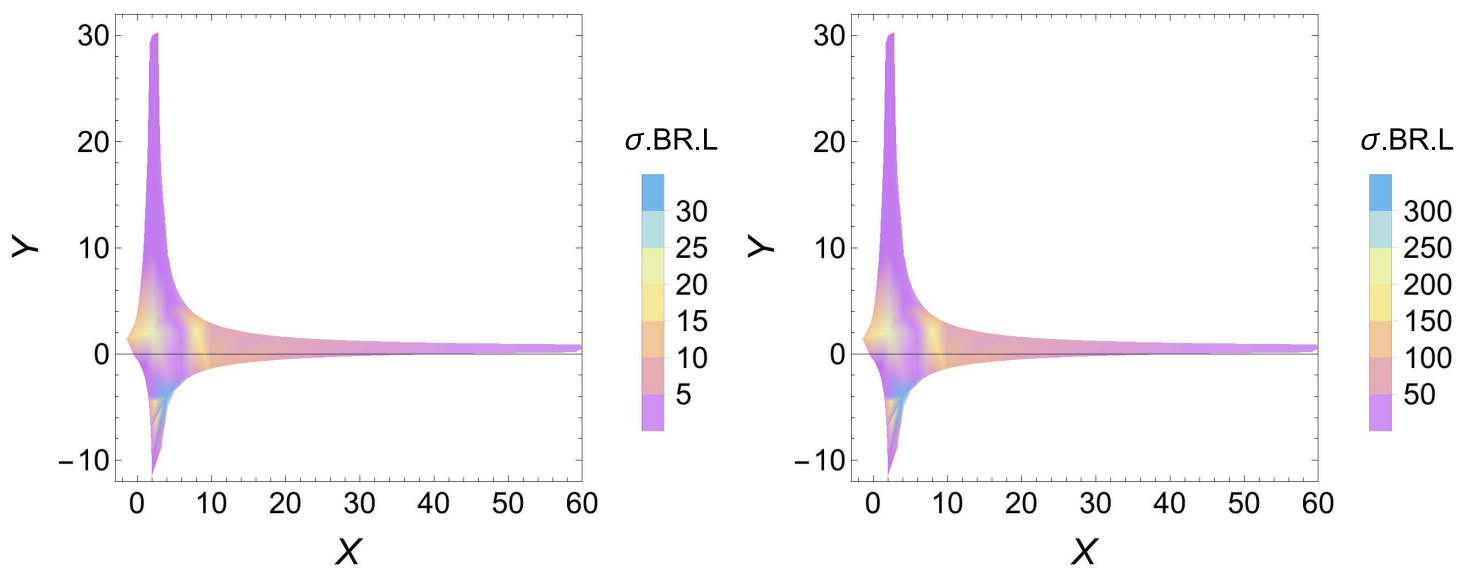

FIG. 7. Event rates ( $\sigma . B R . L)$ at parton level for the neutral Higgs boson $H$, where $L$ is the integrated luminosity. We show Scenario Y for $100 \mathrm{fb}^{-1}$ (left panel) and $1000 \mathrm{fb}^{-1}$ (right panel). We consider $m_{H}=130$.

\begin{tabular}{|c|c|c|c|c|c|c|c|c|c|c|c|}
\hline \hline 2HDM & $X$ & $Y$ & $Z$ & \multicolumn{3}{|c|}{$m_{h}=125 \mathrm{GeV}$} & \multicolumn{2}{|c|}{$m_{H}=130 \mathrm{GeV}$} & \multicolumn{2}{|c|}{$m_{H}=150 \mathrm{GeV}$} & \multicolumn{2}{|c|}{$m_{H}=170 \mathrm{GeV}$} \\
& & & & bs & $\sigma . b s$ & bs & $\sigma . b s$ & bs & $\sigma . b s$ & bs & $\sigma . b s$ \\
\hline \hline Ib35 & 28 & 10 & 28 & 15.66 & 6.392 & 51.8 & 1.209 & 51.6 & 0.30 & 1.58 & 0.117 \\
Ib47 & 30 & 5 & 30 & 16.14 & 3.086 & 48.2 & 10.983 & 48.0 & 0.127 & 1.80 & 0.839 \\
Ib57 & 44 & 5 & 44 & 17.58 & 11.861 & 38.6 & 5.14 & 38.4 & 2.303 & 3.68 & 0.137 \\
\hline IIa11 & 20 & 2 & 20 & 1.42 & 1.055 & 25.2 & 0.097 & 25.0 & 0.091 & 24.8 & 0.085 \\
IIa14 & 26 & 2 & 26 & 1.44 & 1.651 & 26.0 & 0.059 & 25.8 & 0.054 & 25.6 & 0.049 \\
IIa26 & 36 & 1 & 36 & 1.46 & 1.621 & 26.4 & 0.045 & 26.2 & 0.042 & 26.0 & 0.038 \\
\hline Ya11 & 20 & 2 & -2 & 1.42 & 1.084 & 25.2 & 0.062 & 25.0 & 0.059 & 24.8 & 0.054 \\
Ya12 & 22 & 2 & -2 & 1.44 & 1.078 & 25.6 & 0.057 & 25.4 & 0.053 & 25.2 & 0.048 \\
Ya14 & 26 & 2 & -2 & 1.46 & 1.441 & 26.0 & 0.057 & 25.8 & 0.053 & 25.6 & 0.049 \\
\hline \hline
\end{tabular}

TABLE II. Parameters for few optimistic benchmark points in the 2HDM-III as a 2HDM-I, -II and -Y configuration. Here $b s$ stands for $\operatorname{BR}(\phi \rightarrow b \bar{s}+\bar{b} s)$, in units of $10^{-2}$, where $\phi=h, H$, while $\sigma . b s$ stands for the cross section multiplied by the above $\mathrm{BR}$ as obtained at the LHeC in units of $\mathrm{fb}$. We have analyzed only the benchmarks where the $\sigma . b s$ is greater than $0.15 \mathrm{fb}$, so that at least 15 events are produced for $100 \mathrm{fb}^{-1}$.

at this point that these processes and their kinematic features to discover generic Higgs bosons have been studied since long [67, 68]. Further parton level studies have been performed within the SM recently in [69]. In the context of BSM physics, cross section estimates while taking into account Next-to-Leading Order (NLO) correction factors have been performed in [70] but no signal and background studies have been reported therein. In fact, dedicated simulations at the event generator level have not been done extensively and we focus here on these aspects, most importantly, with the intent of detecting two Higgs bosons simultaneously in novel flavor-violating modes.

\section{B. Backgrounds}

There are mainly two groups of SM noise to our Higgs signals. The charged-current backgrounds, $\nu t \bar{b}, \nu b \bar{b} j, \nu b 2 j$, $\nu 3 j$, and the photo-production ones, $e^{-} b \bar{b} j, e^{-} t \bar{t}$. For estimating the cross sections of these SM backgrounds, we have used the same pre-selections like for the signal, Eq. (14), and identical conventions and parameter sets. The expected number of events for $100 \mathrm{fb}^{-1}$ of integrated luminosity are given in the third column of Tab. III]

\section{Signal-to-background analysis}

We passed the CalcHEP v3.4.7 [59] generated parton level event on to PYTHIA v.6.408 [66], which handles the parton shower (both initial and final), hadronization, heavy hadron decays etc. The final state radiation smears the fourmomentum of the jets, thus the invariant mass of the Higgs boson signal is less than the actual values considered in the event. We also took the experimental resolutions of the jet angles and energy using the toy calorimeter PYCELL, 


\begin{tabular}{|c||c|c||c|c|c|c|c|c|c|c|c||c|}
\hline Proc & SimEvt & RawEvt & $\mathrm{a}$ & $\mathrm{b}$ & $\mathrm{c}$ & $\mathrm{d}$ & $\mathrm{e}$ & $\mathrm{f}$ & $\mathrm{g}$ & $\mathrm{h}$ & $\mathrm{i}$ & $\mathcal{S}$ \\
\hline \hline Ib35 & $100 \mathrm{~K}$ & 639.2 & 447.6 & 177.3 & 117.1 & 97.4 & 93.8 & 37.8 & 31.7 & 25.4 & 15.8 & $1.2(3.8)$ \\
Ib47 & $100 \mathrm{~K}$ & 308.6 & 216.8 & 85.1 & 56.2 & 47.1 & 45.5 & 18.4 & 15.6 & 13.0 & 8.1 & $0.62(2.0)$ \\
Ib57 & $100 \mathrm{~K}$ & 1186.1 & 833.7 & 325.7 & 215.5 & 180.6 & 173.9 & 70.3 & 59.1 & 49.3 & 31.1 & $2.4(7.5)$ \\
\hline IIa11 & $100 \mathrm{~K}$ & 105.5 & 74.3 & 29.1 & 19.2 & 16.0 & 15.4 & 6.3 & 5.3 & 4.4 & 2.8 & $0.21(0.70)$ \\
IIa14 & $100 \mathrm{~K}$ & 165.1 & 116.1 & 45.2 & 30.0 & 25.4 & 24.4 & 9.7 & 8.3 & 6.9 & 4.4 & $0.33(1.05)$ \\
IIa26 & $100 \mathrm{~K}$ & 162.1 & 114.4 & 44.7 & 29.5 & 24.5 & 23.6 & 9.5 & 8.1 & 6.8 & 4.3 & $0.33(1.03)$ \\
\hline Ya11 & $100 \mathrm{~K}$ & 108.4 & 76.3 & 29.8 & 19.6 & 16.4 & 15.8 & 6.4 & 5.4 & 4.6 & 2.9 & $0.22(0.70)$ \\
Ya12 & $100 \mathrm{~K}$ & 107.8 & 76.2 & 29.6 & 19.5 & 16.3 & 15.7 & 6.3 & 5.4 & 4.5 & 2.8 & $0.21(0.67)$ \\
Ya14 & $100 \mathrm{~K}$ & 144.1 & 101.7 & 39.8 & 26.0 & 21.7 & 20.8 & 8.2 & 7.0 & 5.9 & 3.8 & $0.29(0.92)$ \\
\hline \hline It $\bar{b}$ & $100 \mathrm{~K}$ & 50712.1 & 28338.4 & 15293.7 & 9845.0 & 8144.2 & 7532.7 & 2982.1 & 2058.0 & 652.2 & 139.6 & \\
$\nu b \bar{b} j$ & $560 \mathrm{~K}$ & 14104.6 & 6122.8 & 3656.7 & 1858.5 & 1787.1 & 1650.1 & 257.5 & 152.5 & 85.2 & 15.1 & \\
$\nu b 2 j$ & $90 \mathrm{~K}$ & 18043.1 & 8389.2 & 3013.0 & 1691.5 & 1445.5 & 1373.7 & 389.5 & 206.1 & 77.2 & 11.3 & $B=170.8$ \\
$\nu 3 j$ & $300 \mathrm{~K}$ & 948064.2 & 410393.4 & 15560.9 & 0.0 & 0.0 & 0.0 & 0.0 & 0.0 & 0.0 & 0.0 & $\sqrt{B}=13.1$ \\
$e b \bar{b} j$ & $115 \mathrm{~K}$ & 256730.1 & 55099.8 & 36353.6 & 12659.8 & 1432.0 & 200.7 & 54.1 & 24.8 & 18.0 & 4.5 & \\
$e t \bar{t}$ & $130 \mathrm{~K}$ & 783.3 & 685.0 & 384.5 & 265.9 & 179.3 & 26.2 & 11.6 & 10.5 & 3.9 & 0.3 & \\
\hline
\end{tabular}

TABLE III. Expected number of events after different combinations of cuts for signal and backgrounds at the LHeC with 100 $\mathrm{fb}^{-1}$ integrated luminosity for $m_{h}=125 \mathrm{GeV}$. SimEvt stands for the actual number of events analyzed in the Monte Carlo simulations. RawEvt stands for the number of events with only the generator-level cuts (14) imposed; for the signal as well as for background, these are calculated from the total cross section times branching ratio. In the final column we mention the significances $(\mathcal{S})$ defined as $\mathcal{S}=S / \sqrt{B}$, where signal events $S$, background events $B$ for $100 \mathrm{fb}^{-1}$ of data after all cuts mentioned in the "i" column. The number in the parenthesis in the final column represent the significances for $1000 \mathrm{fb}^{-1}$.

in accordance with the LHeC detector parameters, given in PYTHIA. This has some non-trivial effect since we used the invariant mass to isolate the Higgs signal. In our study we considered the LHC type calorimeter for the LHeC. Although in reality this is not the case, for example, unlike ATLAS and CMS, the electro-magnetic and the hadronic calorimeter at the $\mathrm{LHeC}$ is not symmetric. However, since we are not doing detector simulation and also we are not considering cracks in the detectors, we applied symmetric large rapidity coverage for jets and leptons in our analysis. We expect that these assumptions hardly alter our findings. The detector parameters in the toy calorimeter module PYCELL are set according to the LHeC detector [62]. Specifically, we assume large calorimeter coverage $|\eta|<5.5$, with segmentation (the number of division in $\eta$ and $\phi$ are 320 and 200 respectively) $\Delta \eta \times \Delta \phi=0.0359 \times 0.0314$. Further, we have used Gaussian energy resolution [60] for both leptons $(\ell=e, \mu)$ and jets (labelled as $j$ ), with

$$
\frac{\Delta E}{E}=\frac{a}{\sqrt{E}} \oplus b
$$

where $a=0.32, b=0.086$ for jets and $a=0.085, b=0.003$ for leptons and $\oplus$ means addition in quadrature. We have used a cone algorithm for the jet-finding algorithm, with jet radius $\Delta R(j)=\sqrt{\Delta \eta^{2}+\Delta \phi^{2}}=0.5$. Calorimeter cells with $E_{T, \text { min }}^{\text {cell }} \geq 5.0 \mathrm{GeV}$ are considered to be potential candidates for jet initiators. All cells with $E_{T, \text { min }}^{\text {cell }} \geq 1.0$ $\mathrm{GeV}$ were treated as part of the would-be jet. A jet is required to have minimum summed $E_{T, \text { min }}^{j} \geq 15 \mathrm{GeV}$ and the jets are ordered in $E_{T}$. Leptons $(\ell=e, \mu)$ are selected if they satisfy the requirements: $E_{T}^{\ell} \geq 15 \mathrm{GeV}$ and $\left|\eta^{\ell}\right| \leq 3.0$. In our jet finding algorithm we include leptons as parts of jets. Finally we separate them, putting some isolation criterion as follows: if we find a jet near a lepton, with $\Delta R(j-\ell) \leq 0.5$ and $0.8 \leq E_{T}^{j} / E_{T}^{\ell} \leq 1.2$, i.e. if the jet $E_{T}$ is nearly identical to that of this lepton, the jet is removed from the list of jets and treated as a lepton. However, if we find a jet within $\Delta R(j-\ell) \leq 0.5$ of a lepton, whose $E_{T}$ differs significantly from that of the lepton, the lepton is removed from the list of leptons. This isolation criterion mostly remove leptons from $b$ or $c$ decays. We reconstructed the missing (transverse) energy $\left(\mathbb{F}_{T}\right)$ from all observed particles and shown in left panel of Fig. 9] We have also calculated the same from the energy deposition in the calorimeter cells and found consistency between these two methods. Only jets with $\left|\eta^{j}\right|<2.5$ and $E_{T}^{j} \geq 15 \mathrm{GeV}$ "matched" with a $b$-flavored hadron ( $B$-hadron), i.e. with $\Delta R(j, B$ - hadron $)<0.2$ is considered to be "taggable". We assume that these jets are actually tagged with probability $\epsilon_{b}=0.50$. We also adopted mis-tagging of non- $b$ jets as $b$-jets and treated $c$-jets differently from the gluon and light-flavor jets. A jet with $\left|\eta^{j}\right| \leq 2.5$ and $E_{T}^{j} \geq 15 \mathrm{GeV}$ matched with a $c$-flavored hadron $(C-$ hadron, e.g., a $D$-meson or $\Lambda_{c}$-baryon), i.e., with $\Delta R(j, C$ - hadron $)<0.2$, is again considered to be taggable, with (mis)tagging probability $\epsilon_{c}=0.10$. Jets that are associated with a $\tau$-lepton, with $\Delta R(j, \tau) \leq 0.2$, and all jets with $\left|\eta^{j}\right|>2.5$, are taken to have vanishing tagging probability. All other jets with $E_{T}^{j} \geq 15 \mathrm{GeV}$ and $\left|\eta^{j}\right| \leq 2.5$ are assumed to be (mis-)tagged with probability $\epsilon_{u, d, s, g}=0.01$. These efficiencies follow recent LHeC analyses $[69]$.

The analysis strategy has been adopted from earlier work of some of us [71]. In particular, we have exploited a 


\begin{tabular}{|c||c|c||c|c|c|c|c|c|c|c|c||c|}
\hline Proc & SimEvt & RawEvt & $\mathrm{A}$ & $\mathrm{B}$ & $\mathrm{C}$ & $\mathrm{D}$ & $\mathrm{E}$ & $\mathrm{F}$ & $\mathrm{G}$ & $\mathrm{H}$ & $\mathrm{I}$ & $\mathcal{S}$ \\
\hline \hline Ib35 & $100 \mathrm{~K}$ & 120.9 & 87.1 & 34.1 & 26.9 & 22.5 & 21.6 & 7.5 & 6.1 & 5.3 & 3.4 & $0.28(0.88)$ \\
Ib47 & $100 \mathrm{~K}$ & 1098.3 & 790.3 & 307.1 & 243.9 & 204.6 & 195.7 & 68.5 & 56.1 & 48.6 & 31.3 & $2.6(8.1)$ \\
Ib57 & $100 \mathrm{~K}$ & 514.0 & 371.2 & 144.8 & 115.0 & 96.0 & 92.0 & 31.7 & 25.8 & 22.7 & 14.3 & $1.2(3.7)$ \\
\hline IIa11 & $100 \mathrm{~K}$ & 9.7 & 6.8 & 2.7 & 2.1 & 1.8 & 1.7 & 0.6 & 0.4 & 0.3 & 0.2 & $0.02(0.05)$ \\
IIa14 & $100 \mathrm{~K}$ & 5.9 & 4.2 & 1.7 & 1.3 & 1.1 & 1.0 & 0.4 & 0.3 & 0.2 & 0.1 & $0.01(0.02)$ \\
IIa26 & $100 \mathrm{~K}$ & 4.5 & 3.1 & 1.3 & 1.0 & 0.8 & 0.8 & 0.3 & 0.2 & 0.1 & 0.1 & $0.01(0.02)$ \\
\hline Ya11 & $100 \mathrm{~K}$ & 6.2 & 4.4 & 1.8 & 1.4 & 1.1 & 1.1 & 0.4 & 0.3 & 0.2 & 0.1 & $0.01(0.02)$ \\
Ya12 & $100 \mathrm{~K}$ & 5.7 & 4.0 & 1.6 & 1.3 & 1.0 & 1.0 & 0.3 & 0.2 & 0.2 & 0.1 & $0.01(0.02)$ \\
Ya14 & $100 \mathrm{~K}$ & 5.7 & 4.0 & 1.6 & 1.3 & 1.0 & 1.0 & 0.3 & 0.2 & 0.2 & 0.1 & $0.01(0.02)$ \\
\hline \hline$\nu t \bar{b}$ & $100 \mathrm{~K}$ & 50712.1 & 28338.4 & 15293.7 & 10976.4 & 9092.4 & 8393.6 & 2550.9 & 1565.5 & 617.9 & 113.7 & \\
$\nu b \bar{b} j$ & $560 \mathrm{~K}$ & 14104.6 & 6122.8 & 3656.7 & 2145.5 & 2062.1 & 1902.9 & 266.6 & 141.0 & 87.5 & 14.4 & \\
$\nu b 2 j$ & $90 \mathrm{~K}$ & 18043.1 & 8389.2 & 3013.0 & 2053.6 & 1734.0 & 1650.1 & 402.8 & 143.7 & 64.5 & 8.1 & $B=147.8$ \\
$\nu 3 j$ & $300 \mathrm{~K}$ & 948064.2 & 410393.4 & 15560.9 & 0.0 & 0.0 & 0.0 & 0.0 & 0.0 & 0.0 & 0.0 & $\sqrt{B}=12.2$ \\
$e b \bar{b} j$ & $115 \mathrm{~K}$ & 256730.1 & 55099.8 & 36353.6 & 16838.4 & 1826.6 & 284.1 & 56.4 & 31.6 & 22.6 & 11.3 & \\
$e t \bar{t}$ & $130 \mathrm{~K}$ & 783.3 & 685.0 & 384.5 & 280.8 & 190.8 & 27.8 & 10.9 & 9.3 & 3.9 & 0.3 & \\
\hline
\end{tabular}

TABLE IV. Same as Tab. III but for $m_{H}=130 \mathrm{GeV}$. The criterion for jets and $b$-tagging are the same, so that the number of events in column $A$ and $B$ are the same for all SM backgrounds.

\begin{tabular}{|c||c|c||c|c|c|c|c|c|c|c|c||c|}
\hline Proc & SimEvt & RawEvt & A & B & C & D & E & F & G & H & I & $\mathcal{S}$ \\
\hline \hline Ib35 & $100 \mathrm{~K}$ & 30.0 & 23.3 & 9.1 & 8.2 & 6.9 & 6.5 & 1.5 & 1.3 & 1.2 & 0.8 & $0.10(0.33)$ \\
Ib47 & $100 \mathrm{~K}$ & 12.7 & 9.9 & 3.8 & 3.4 & 2.9 & 2.7 & 0.6 & 0.5 & 0.5 & 0.3 & $0.04(0.12)$ \\
Ib57 & $100 \mathrm{~K}$ & 230.3 & 179.6 & 69.3 & 62.6 & 52.6 & 49.9 & 11.7 & 10.1 & 9.1 & 6.4 & $0.83(2.62)$ \\
\hline IIa11 & $100 \mathrm{~K}$ & 9.1 & 6.9 & 2.7 & 2.4 & 2.0 & 1.9 & 0.4 & 0.4 & 0.3 & 0.2 & $0.026(0.08)$ \\
IIa14 & $100 \mathrm{~K}$ & 5.4 & 4.1 & 1.6 & 1.4 & 1.2 & 1.1 & 0.3 & 0.2 & 0.2 & 0.1 & $0.013(0.04)$ \\
IIa26 & $100 \mathrm{~K}$ & 4.2 & 3.2 & 1.3 & 1.1 & 0.9 & 0.9 & 0.2 & 0.1 & 0.1 & 0.1 & $0.013(0.04)$ \\
\hline Ya11 & $100 \mathrm{~K}$ & 5.9 & 4.5 & 1.8 & 1.6 & 1.3 & 1.2 & 0.3 & 0.2 & 0.2 & 0.1 & $0.013(0.04)$ \\
Ya12 & $100 \mathrm{~K}$ & 5.3 & 4.0 & 1.6 & 1.4 & 1.2 & 1.1 & 0.3 & 0.2 & 0.2 & 0.1 & $0.013(0.04)$ \\
Ya14 & $100 \mathrm{~K}$ & 5.3 & 4.0 & 1.6 & 1.4 & 1.2 & 1.1 & 0.3 & 0.2 & 0.2 & 0.1 & $0.013(0.04)$ \\
\hline \hline$\nu t \bar{b}$ & $100 \mathrm{~K}$ & 50712.1 & 28338.4 & 15293.7 & 11810.9 & 9808.7 & 9039.0 & 751.7 & 476.8 & 194.5 & 32.3 & \\
$\nu b \bar{b} j$ & $560 \mathrm{~K}$ & 14104.6 & 6122.8 & 3656.7 & 2395.6 & 2300.1 & 2120.8 & 199.3 & 112.4 & 70.8 & 12.4 & \\
$\nu b 2 j$ & $90 \mathrm{~K}$ & 18043.1 & 8389.2 & 3013.0 & 2427.2 & 2030.3 & 1933.1 & 234.2 & 83.7 & 41.0 & 6.3 & $B=60.1$ \\
$\nu 3 j$ & $300 \mathrm{~K}$ & 948064.2 & 410393.4 & 15560.9 & 0.0 & 0.0 & 0.0 & 0.0 & 0.0 & 0.0 & 0.0 & $\sqrt{B}=7.7$ \\
$e b \bar{b} j$ & $115 \mathrm{~K}$ & 256730.1 & 55099.8 & 36353.6 & 21280.9 & 2270.8 & 385.6 & 36.1 & 24.8 & 20.3 & 9.0 & \\
$e t \bar{t}$ & $130 \mathrm{~K}$ & 783.3 & 685.0 & 384.5 & 291.5 & 199.0 & 29.1 & 3.5 & 3.0 & 1.2 & 0.1 & \\
\hline
\end{tabular}

TABLE V. Same as Tab. IV but for $m_{H}=150 \mathrm{GeV}$.

simple cut-based method for signal enhancement and background rejection. We have chosen the following selections and applied them cumulatively for the signal from $h(H)$.

- $\mathbf{a}(\mathbf{A})$ : We first selected events containing at least three jets (same). The distribution of the number of jet $\left(N_{j e t}\right)$ is shown in the left panel of Fig. 8. For the lighter Higgs, $h$, in all the signal benchmarks the efficiencies 8 are approximately $70 \%$ (For heavier Higgs boson $H$, with mass of 130,150 and $170 \mathrm{GeV}$ the efficiencies are $71 \%$, $76 \% 81 \%$ respectively, as two of the jets are directly coming from the corresponding heavier Higgs boson and hence the efficiencies are larger.). Further, $t \bar{b}$ has efficiency of approximately $56 \%, 2 b j$ and $3 j$ events display approximately $45 \%$ whereas the $b 2 j$ one has approximately $47 \%$. Efficiencies are generally lower for the photoproduction channels: $e 2 b j$ is approximately $20 \%$ (the sharp fall is due to the isolation criterion) whereas for $e 2 t$ the jet efficiencies are higher and due to the presence of two $t$-quarks leads to two $b$-quarks and the probability of having two jets from $W$-boson decay itself is approximately $91 \%$, thus, out of 4 -jets in $91 \%$ events, the probability of having at least three energetic jets is reduced by $4 \%$, which leads to approximately $87 \%$ efficiency. However, as we will see, the presence of the electron in the photo-production modes leads to the corresponding backgrounds to be very low.

\footnotetext{
8 Unless mentioned otherwise, all the efficiencies quoted hereafter are given with respect to the previous selection.
} 


\begin{tabular}{|c||c|c||c|c|c|c|c|c|c|c|c||c|}
\hline Proc & SimEvt & RawEvt & $\mathrm{A}$ & $\mathrm{B}$ & $\mathrm{C}$ & $\mathrm{D}$ & $\mathrm{E}$ & $\mathrm{F}$ & $\mathrm{G}$ & $\mathrm{H}$ & $\mathrm{I}$ & $\mathcal{S}$ \\
\hline \hline Ib35 & $100 \mathrm{~K}$ & 11.7 & 9.6 & 3.7 & 3.5 & 3.0 & 2.8 & 0.5 & 0.4 & 0.4 & 0.3 & $0.053(0.17)$ \\
Ib47 & $100 \mathrm{~K}$ & 83.9 & 69.2 & 26.7 & 25.5 & 21.5 & 20.2 & 3.6 & 3.1 & 3.0 & 2.2 & $0.39(1.23)$ \\
Ib57 & $100 \mathrm{~K}$ & 13.7 & 11.2 & 4.3 & 4.1 & 3.4 & 3.2 & 0.6 & 0.5 & 0.5 & 0.4 & $0.07(0.22)$ \\
\hline IIa11 & $100 \mathrm{~K}$ & 8.5 & 7.0 & 2.7 & 2.5 & 2.1 & 2.0 & 0.3 & 0.3 & 0.3 & 0.2 & $0.035(0.11)$ \\
IIa14 & $100 \mathrm{~K}$ & 4.9 & 4.1 & 1.6 & 1.5 & 1.3 & 1.2 & 0.3 & 0.17 & 0.16 & 0.12 & $0.021(0.07)$ \\
IIa26 & $100 \mathrm{~K}$ & 3.8 & 3.1 & 1.2 & 1.1 & 0.9 & 0.9 & 0.1 & 0.1 & 0.1 & 0.1 & $0.02(0.06)$ \\
\hline Ya11 & $100 \mathrm{~K}$ & 5.4 & 4.4 & 1.7 & 1.6 & 1.4 & 1.3 & 0.2 & 0.2 & 0.2 & 0.1 & $0.02(0.06)$ \\
Ya12 & $100 \mathrm{~K}$ & 4.8 & 4.0 & 1.5 & 1.4 & 1.2 & 1.1 & 0.2 & 0.2 & 0.2 & 0.1 & $0.02(0.06)$ \\
Ya14 & $100 \mathrm{~K}$ & 4.9 & 4.0 & 1.6 & 1.5 & 1.2 & 1.1 & 0.2 & 0.2 & 0.1 & 0.1 & $0.02(0.06)$ \\
\hline \hline$\nu t \bar{b}$ & $100 \mathrm{~K}$ & 50712.1 & 28338.4 & 15293.7 & 12381.7 & 10299.7 & 9465.2 & 209.7 & 144.5 & 75.9 & 13.2 & \\
$\nu b \bar{b} j$ & $560 \mathrm{~K}$ & 14104.6 & 6122.8 & 3656.7 & 2568.2 & 2465.8 & 2272.4 & 103.7 & 60.8 & 37.4 & 8.7 & \\
$\nu b 2 j$ & $90 \mathrm{~K}$ & 18043.1 & 8389.2 & 3013.0 & 2744.8 & 2278.1 & 2171.4 & 99.5 & 40.0 & 25.2 & 5.3 & $B=31.7$ \\
$\nu 3 j$ & $300 \mathrm{~K}$ & 948064.2 & 410393.4 & 15560.9 & 0.0 & 0.0 & 0.0 & 0.0 & 0.0 & 0.0 & 0.0 & $\sqrt{B}=5.6$ \\
$e b \bar{b} j$ & $115 \mathrm{~K}$ & 256730.1 & 55099.8 & 36353.6 & 25010.7 & 2638.4 & 453.3 & 29.3 & 18.0 & 11.3 & 4.5 & \\
$e t \bar{t}$ & $130 \mathrm{~K}$ & 783.3 & 685.0 & 384.5 & 298.8 & 204.5 & 29.9 & 1.0 & 0.8 & 0.5 & 0.0 & \\
\hline
\end{tabular}

TABLE VI. Same as Tab. IV but for $m_{H}=170 \mathrm{GeV}$.
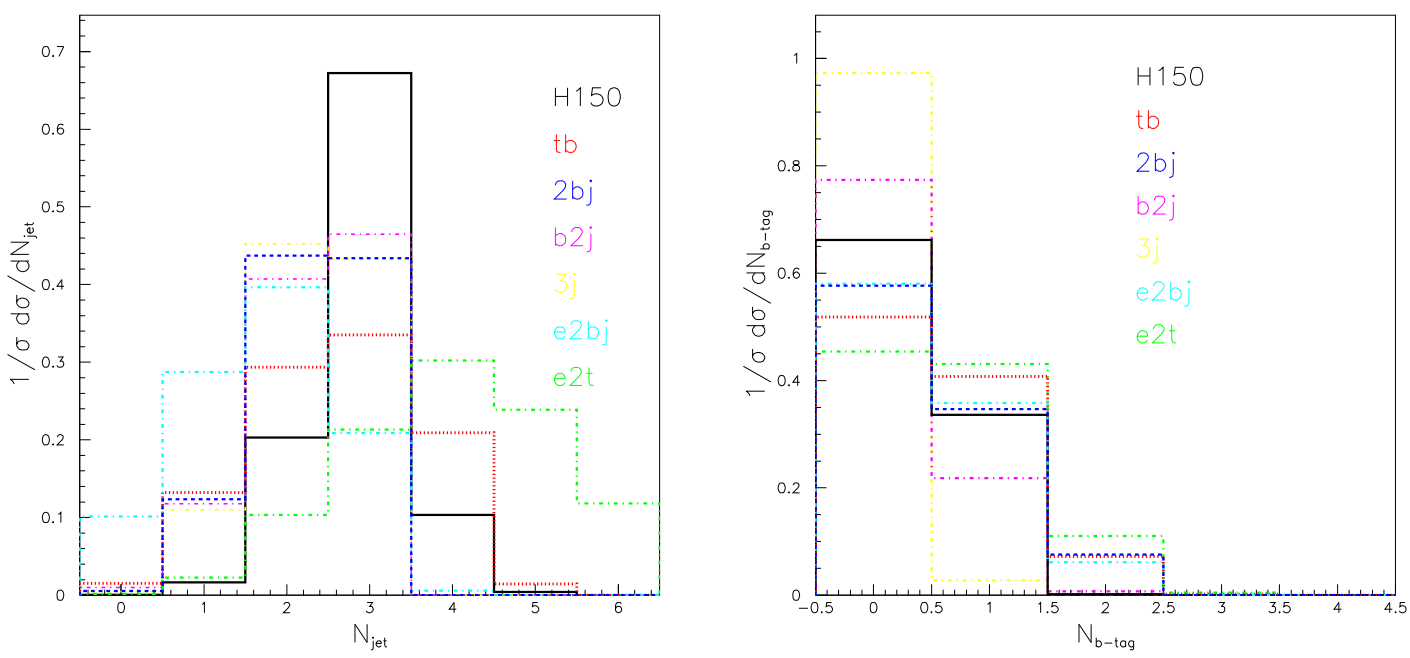

FIG. 8. Scenario Ib with the parameter $X=28=Z$ and $Y=10$. The number of jets $\left(N_{\text {jet }}\right)$ in the left panel and the number of $b$-tagged jet with the inclusion of mis-tagging $\left(N_{b-t a g}\right)$ in the right panel for signal $\left(m_{H}=150 \mathrm{GeV}\right)$ and all the SM backgrounds. For other signal events, the distribution profiles are very similar, except the fact that the number of jets as well as $b$-tagged jets is slightly larger for heavy Higgs bosons. See the fourth(fifth) column for their actual efficiencies with selections applied in $\mathrm{a}(\mathrm{b})$ and $\mathrm{A}(\mathrm{B})$ for lighter(heavier) Higgs bosons, respectively.

- $\mathbf{b}(\mathbf{B})$ : We demanded at least one $b$-tagged jet with the inclusion of proper mis-tagging (same). The distributions of the number of $b$-tagged jets $\left(N_{b-t a g}\right)$ are shown in the right panel of Fig. 8 . For the lighter Higgs, $h$, in all the signal benchmarks the $b$-tagging efficiencies are approximately $40 \%$. In fact, all our signal benchmarks contain at least one $b$-quark and, since we adopted $\epsilon_{b}=0.50$, the $10 \%$ lowering is quite realistic and due to the fact that not all $b$-quarks in the signal are eligible for the $b$-taggable criterion adopted in our analysis. For heavier Higgs signals, benchmarks show similar efficiencies and changes are less than $1 \%$ for three masses. In case of $t \bar{b}$, the events containing at least one $b$-tagged jet are approximately $54 \%$, a rate greater than the signal ones, as these background topologies contain at least two $b$-quarks at the parton level so that, due to combinatorics (other than mis-tagging a light-flavor quark-jet from $W$-boson decays), the probability of one $b$-tag is more. The probability of $2 b j$ is approximately $60 \%$, approximately $6 \%$ larger than $t \bar{b}$, due to, unlike $t \bar{b}$, the presence of one $b$-quark and one light-flavor jet in the hard processes. Further, $b 2 j$ efficiencies are similar to or little less than those of the signals due to the fact that the taggable rapidity is more central, where the jets are more likely to be forward in the basic hard processes. The efficiency of $e 2 t$ is approximately $2 \%$ larger than for $t \bar{b}$ mainly due 
to the contributions from mis-tagging one extra hadronic $W$, i.e, two extra jets. Finally, the efficiency of $3 j$ is approximately $4 \%$, which seems consistent with the expected mis-tagging rates (1.0\% for light-flavor jets and $10 \%$ for $c$-jets) in presence of combinatorics.

- $\mathbf{c}(\mathbf{C})$ : We demanded at least two central jets, with $p_{T}>30 \mathrm{GeV}\left(p_{T}>25,20\right.$ and $15 \mathrm{GeV}$ for $m_{H}=130,150$ and $170 \mathrm{GeV}$, respectively 9 ) in the pseudo-rapidity range $|\eta|<2.5$. One of the central jet must be a $b$-tagged jet and we demanded only one $b$-tagged jet (same). For the lighter Higgs, $h$, all the signal benchmarks as well as the $t \bar{b}$ noise survive at the rate of approximately $65 \%$ since all these processes naturally have three jet in their events. Further, $e 2 b j$ and $2 b j$ are reduced by approximately $35 \%$ and $50 \%$, respectively, mainly due to demanding, with respect to the $\mathrm{b}(\mathrm{B})$ case above, of one $b$-tagged jet only. The diagrams of $t b$ and $e 2 t$ reveal that these two backgrounds are more central, because of the presence of one $t$-quark in the central region. In fact, the efficiency is larger in $e 2 t$ and is mainly due to the contributions from the additional $t$-quark. Although in $2 b j$ the probability in presence of one $b$-tagged in the central region is large the overall efficiency is reduced to $12 \%$ due to (partly) the possibility of more than one $b$-tagged jets whereas for $W b$ the value is mainly due to the hadronic branching fraction and also that the $b$-tagged jet is not necessary central. In case of $e 2 b j$ the efficiency is $35 \%$ : this noise suffers mainly due to the centrality criterion. For $3 j$, none of the events survived this selection criterion. The efficiencies pattern discussed above are similar for the heavier Higgs boson, $H$, yet recall that here we have used slightly softer selections on the transverse momentum. Thus, the efficiencies are increasing with a softer $p_{T}$ selection for both signals and backgrounds.

- $\mathbf{d}(\mathbf{D})$ : The missing transverse energy cut $\mathbb{H}_{T}>20 \mathrm{GeV}$ is first applied (same). The relevant distribution is shown in the left panel of Fig. 9, For all the signal benchmarks (lighter as well heavier Higgs bosons), $t \bar{b}$ and $b 2 j$
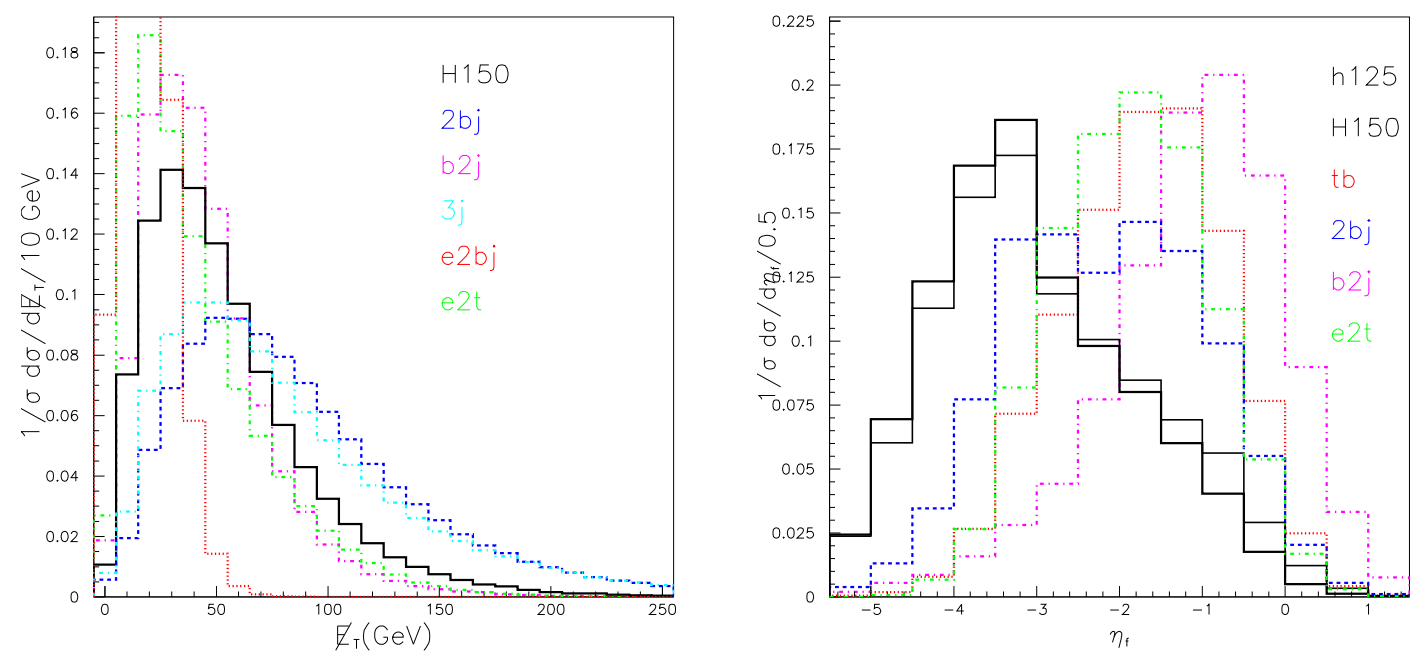

FIG. 9. The missing energy $\left(\not_{T}\right)$ (left panel) and rapidity $\left(\eta_{j_{f}}\right)$ (right panel) profile of the forward jet for signals and SM backgrounds. The $\mathbb{E}_{T}$ distributions for all other signal benchmarks as well as the $t \bar{b}$ noise are not shown as they are very similar to the signal distributions of $m_{H}=150 \mathrm{GeV}$ for Scenario Ib with $X=Z=28$ and $Y=10$ (shown in thick solid), whereas the thin solid is for $m_{h}=125 \mathrm{GeV}$ for Scenario Ia with $X=Z=28$ with $Y=10$. The rapidity distributions profile for $m_{H}=130(170) \mathrm{GeV}$ is very close to the $m_{h}=125 \mathrm{GeV}\left(m_{H}=130 \mathrm{GeV}\right)$ case shown in thin solid, except that for massive Higgs the peaks shift towards the left. Also the corresponding rapidity distribution profile for $e 2 b j$ is somewhat similar to the $m_{h}=125 \mathrm{GeV}$ signal case.

the efficiencies are approximately $83 \%$. The sources of neutrinos and the event structures of these two processes are very similar, except for the fact that the top-quark decays produce either neutrinos (which are then pure sources of missing energy but in such a case, owing to the selection a(A), they are largely removed) or quarks (where the smearing of jets and track mis-measurements are the main sources of missing energy). For $2 b j$ the efficiencies are approximately $96 \%$. This selection is crucial to suppress the photo-production processes: $e 2 b j$ and $e 2 t$. In case of $e 2 b j$ only $12 \%$ of the events survive in fact. For $e 2 t$ the presence of two $W$-bosons and their

9 Since the cross sections become smaller with increasing $m_{H}$, we lowered the central jet $p_{T}$ cuts. 
decays into leptonic modes would be the sources of missing energy, so that this noise is not affected very much by our constraints.

- $\mathbf{e}(\mathbf{E})$ : A lepton $\left(e\right.$ or $\mu$ ) veto for $p_{T}>20 \mathrm{GeV}$ and $\eta<3.0$ is applied here (same). For the lighter Higgs signal benchmarks, the efficiency for this selection is approximately $96 \%$, as only $4 \%$ of the events contain at least one lepton which is coming from the semileptonic decays of $B$-hadrons and $D$-mesons and which passes the isolation criterion above. The efficiencies for $2 b j$ and $b 2 j$ are rather close to the signal benchmarks, due to these processes also not having prompt leptons in their events. The $t \bar{b}$ channel has an efficiency of $93 \%$, hence approximately $3 \%$ less than the signal, as here the top-quark decays can lead to one bottom-quark and, if the hard-processes bottom quarks are more central, the requirement in $\mathrm{c}(\mathrm{C})$ is satisfied and there is no problem in having a central and high- $p_{T}$ lepton from a $W$-boson decay. In the photo-production processes, $e 2 b j$ and $e 2 t$ both contain hard leptons, so only $15 \%$ of the events survive our lepton veto. For heavier Higgs masses, the efficiencies are quite similar to the lighter Higgs boson mass case. The only difference is that, since the applied $p_{T}$ threshold is lower for heavier Higgs masses, the probability of having a lepton in the event is higher, thus the veto efficiencies are somewhat smaller, except for the two photo-production processes.

- $\mathbf{f}(\mathbf{F})$ : In the central region, defined above via $\mathrm{c}(\mathrm{C})$, we reconstruct the invariant mass of one $b$-tagged jet with any of the other jets, $M_{b j}$. Amongst these, we have chosen the best combination, i.e., where, the absolute difference $\left|M_{b j}-M_{h(H)}\right|$ is minimized. We call this di-jet combination the candidate light (heavy) Higgs boson signal. In order to select the latter, we have kept events within a $15 \mathrm{GeV}$ mass window centered around the corresponding Higgs boson masses. The distributions of $M_{b j}$ are shown in the left panel of Fig. 10, It seems that the di-jet invariant masses of the BP-Ia30 signal benchmark (with $m_{h}=125 \mathrm{GeV}$ ) has its peak around $115 \mathrm{GeV}$. The distribution for $t \bar{b}$ also has a peak around that of the $m_{h}=125 \mathrm{GeV}$ signal. However, the combinatorics is significant and this shows in their efficiencies, which are approximately $40 \%$ for both. The distribution of $b 2 j$ is flat as there is no correlations for the correct di-jet candidates. Also note that $b 2 j$ has $W$-boson exchange resonant diagrams, so a probability in principle exists for a di-jet invariant mass peak at $M_{W}$, however, this is very small, mainly due to low mis-tagging efficiencies and the centrality criterion. Further, also in case of $2 b j$, where the $Z$-boson is present resonantly in the diagrams, the $Z \rightarrow b \bar{b}$ decay combined with high tagging efficiencies allows for the appearance of a di-jet peak at $80 \mathrm{GeV}$ (approximately $10 \mathrm{GeV}$ less than $M_{Z}$ due to jet energy smearing): see the left-panel of Fig. 10. In case of $e 2 t$, like $t \bar{b}$, one has also correlated di-jet candidates, but the energy scale is higher, so the peak is shifted to higher masses. The efficiency is approximately $45 \%$, a little larger than the signal and $t \bar{b}$ ones. The distributions of $b 2 j$ and $e 2 b j$ are flat and the efficiencies are the same, approximately $28 \%$. This particular selection suppresses $2 b j$ events more severely though, at a rate which is approximately 15\%. For heavier Higgs bosons the distributions show rapid falls and so, by applying the mass window cuts, only the left part of the distributions contributes. This shows in their signal efficiencies, which are approximately $34 \%, 23 \%$ and $18 \%$ for $m_{H}=130,150$ and $170 \mathrm{GeV}$, respectively. The SM backgrounds do not show up in distributions at large invariant masses, thus for heavier Higgs mass combined with the same mass window selection suppresses more the backgrounds. As an example, in case of $e 2 t$, which produces somewhat higher invariant masses than all other SM backgrounds, the efficiencies drops to 40\%, $12 \%$ and $4 \%$ for $m_{H}=130$, 150 and $170 \mathrm{GeV}$, respectively. In case of $t \bar{b}$ the efficiencies (see Tabs. III IV [V] and VI) drop to from $30 \%$, $8 \%$ and $2 \%$, respectively. In case of $2 b j$ the values are $14 \%, 10 \%$ and $5 \%$. For $e 2 b j$, one has $20 \%, 10 \%$ and $7 \%$, respectively. Finally, for $b 2 j$, these are $25 \%, 12 \%$ and $5 \%$, respectively.

- $\mathbf{g}(\mathbf{G})$ : We demanded the remaining leading jet in the event to have $p_{T}>25 \mathrm{GeV}$, with $-5.5<\eta<-0.5(-1.0)$ (these values are chosen by seeing the distribution, see the right panel of Fig. 9) and termed it as the forward jet $\left(j_{f}\right)$. This forward jet lies very close to the direction of the incoming proton, like in Vector Boson Fusion (VBF) processes for Higgs production. In contrast to VBF though, instead of a jet with large rapidity gap with respect the the forward jet (a backward jet then), in our signal we have a neutrino. The more massive the Higgs is, the less energy remains for the forward jet so as to lay close to the proton direction, i.e., at larger rapidity. This reflects in the right panel of Fig. 9. The thick (thin) solid curve corresponds to $m_{H}=150 \mathrm{GeV}\left(m_{h}=125\right.$ $\mathrm{GeV}$ ). For a lighter Higgs boson, $m_{h}=125 \mathrm{GeV}$, the efficiency is approximately $80 \%$. For $e 2 t$ the efficiency is almost $90 \%$, twice that of $e 2 b j$, as there is more than twice a probability to have a forward-jet from top-quark decays.

- $\mathbf{h}(\mathbf{H})$ : The di-jet invariant mass of the Higgs boson candidates with the forward tagged jet, which is essentially the overall energy scale of the hard scattering, is asked to comply with the following requirements: $m_{h j_{f}}\left(m_{H j_{f}}\right)$

10 The peaks always show up to the left side of the actual masses due to jet energy smearing and the shift also depends on the jet-cone size. 

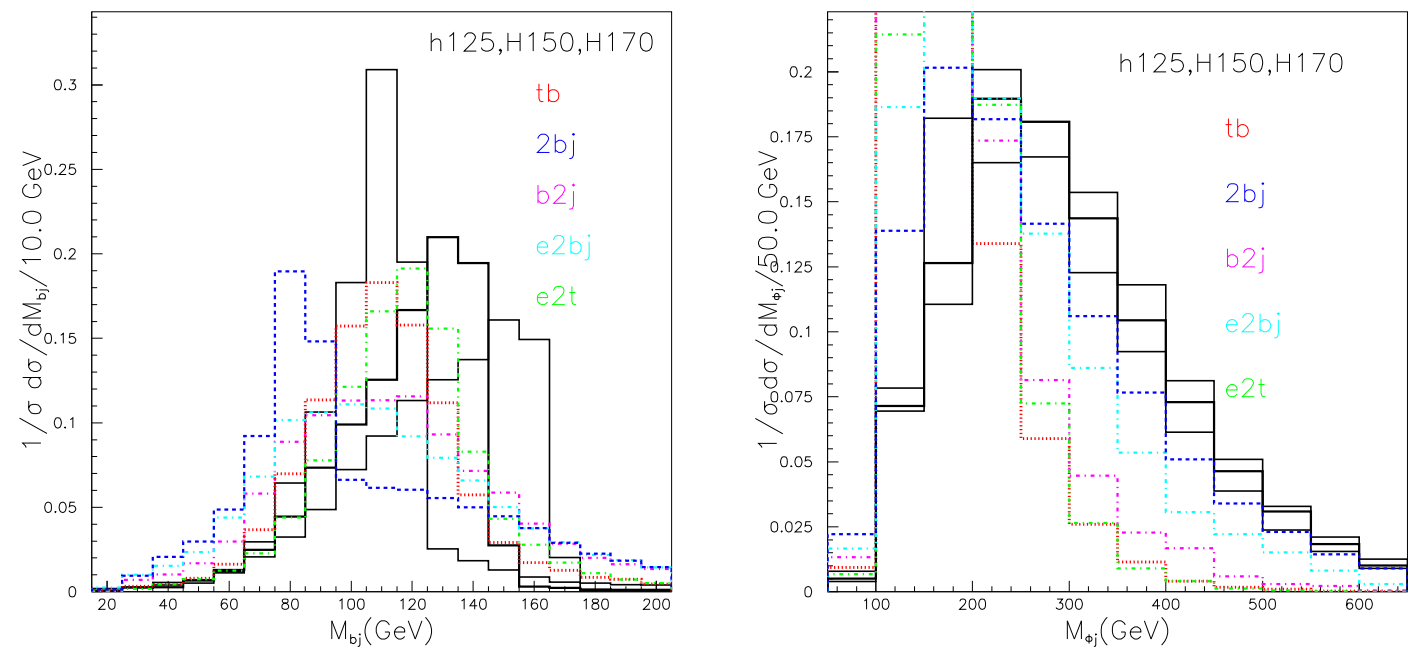

FIG. 10. The di-jet invariant mass, made up by one $b$-tagged and one light-flavor jet, producing Higgs candidates, $M_{\phi}=M_{b j}$ (left panel) and the three-jet invariant mass, i.e., the previous two jets combined together with the forward jet, $M_{\phi j_{f}}$ (right panel). The mass peaks of the Higgs signals $\left(M_{\phi}\right)$ correspond to $m_{h}=125$ (thin black) for Scenario Ia, $m_{H}=150$ (thick black) and 170 (thin black) for Scenario Ib from left to right. All these are using the parameters $X=Z=28$ and $Y=10$. The distribution for $m_{H}=130$ is not shown but it lies in between $m_{h}=125$ and $m_{H}=150$. Among all SM backgrounds, only $2 b j$ shows a prominent peak from the $Z$-boson. Notice that $M_{\phi j_{f}}$ represents the overall energy scale of the hard-scattering.

$>190 \mathrm{GeV}$ (190, 210 and $230 \mathrm{GeV}$ for $m_{H}=130,150$ and $170 \mathrm{GeV}$, respectively). The distributions are shown in the right panel of Fig. 10. For a lighter Higgs boson, $m_{h}=125 \mathrm{GeV}$, except a few cases 11 , the efficiency is approximately $82 \%$. This forward jet should not be a $b$-jet though. So, in $t \bar{b}$ and $e 2 t$, where a forward $b$-tag jet is more probable, the efficiencies are lower, approximately $32 \%$ and $37 \%$. It is clear from the right panel of Fig. 10 that the three-jet invariant mass distributions of $b 2 j$ peak around $140 \mathrm{GeV}$ or so. The same for $t \bar{b}, e 2 t, 2 b j$, $e 2 b j$ and the Higgs signal with $m_{h}=125 \mathrm{GeV}$, which show somewhere around $180 \mathrm{GeV}$. So, for $m_{h}=125 \mathrm{GeV}$, the efficiency is around $82 \%$. For the heavier Higgs boson, with $m_{H}=150$ (170) GeV, the distributions are shown in the thick (thin) solid curve in the right panel of Fig. 10 and peak around 220 (260) GeV. The selection cuts for these two Higgs bosons are 210 and $230 \mathrm{GeV}$, respectively. With our selection, for these two heavy Higgs signals, one suppresses more SM backgrounds than in the case of the Higgs signal with $m_{h}=125 \mathrm{GeV}$ and $m_{H}=130 \mathrm{GeV}$. For example, in the $t \bar{b}$ case, the most dominant background, for $m_{h}=125 \mathrm{GeV}$, and $m_{H}=130$, 150 and170 GeV, the events which survived are approximately 652, 618, 195 and 80, respectively. For other SM backgrounds a similar pattern follows, thus the overall SM backgrounds can be strongly reduced. However, this overall background rejection will not help alone to have larger significances as the signal rate itself is suppressed with heavier Higgs masses (at the production level).

- i(I): Finally, we required only one light-flavor jet in the central region (same). This selection is called 'central jet veto' and has severe impact on all processes having more jets in the central rapidity region, other than the Higgs candidate jets. Recall that our Higgs signal candidate jets, selected in $\mathrm{f}(\mathrm{F})$ above, are central: this is true for not only the signal, but also the dominant SM background, $t \bar{b}$. For a lighter SM Higgs, see Tab. [II] approximately 35-40\% of the events have a central jet other than Higgs candidate jets, thus only $60-65 \%$ of the events survive. For $t \bar{b}, \nu b 2 j$ and $\nu 2 b j$ the efficiencies are $22 \%, 18 \%$ and $14 \%$, respectively. Among all the SM backgrounds, $e 2 t$ has a larger number of jets (see the distributions in the left panel of Fig. 8), thus the probability of having a central jet is more, so that this selection suppresses this background severely, approximately by $93 \%$ (for all the Higgs cases, see Tabs. III IV [V] and VI).

After the cumulative selections from a-i, discussed above, we find that, for the SM Higgs boson with $m_{h}=125$, the final number of events is around 15-30 only for Scenario Ib and for large values of the parameters $X=Z=28(44)$

11 Recall that the efficiencies are relative to the previous selection, one can estimate the individual efficiencies from the respective distributions. 
and $Y=10(5)$ respectively. The total SM background rate is approximately 170 . The charged-current backgrounds, $\nu t \bar{b}, \nu b \bar{b} j$ and $\nu b 2 j$, are the dominant ones and only $3 \%$ of the total background comes from et $\bar{t}$ photo-production. These rates lead to a maximum significance of approximately $2.4(7.5) \sigma$ with $100(1000) \mathrm{fb}^{-1}$ integrated luminosity for Scenario Ib with $X=Z=44$ and $Y=5$. For Scenario Ib with $X=Z=28$ and $Y=10$, the significance is approximately $1.2(3.8) \sigma$ for $100(1000) \mathrm{fb}^{-1}$ integrated luminosity. The significances for Scenario Y and Scenario IIa are less than 1. Thus, one can expect that Scenario Ib with large value of $X=44=Z$ and $Y=5$ may be accessible through the SM-like Higgs boson signal already detected at the LHC.

We also searched for the second CP-even neutral Higgs boson of our 2HDM-III, with masses $m_{H}=130$, 150 and $170 \mathrm{GeV}$. After the cumulative selections from A-I, the maximum number of signal events for $m_{H}=130 \mathrm{GeV}$ is approximately 15 (30) and only for Scenario Ib with $X=Z=44$ (30) with $Y=5$. The total SM background is approximately 150 . So the maximum significance is approximately $2.6(8.1) \sigma$ for $100(1000) f^{-1}$ integrated luminosity. For the case $m_{H}=150 \mathrm{GeV}$, the number of signal events is approximately 7 and the SM background reduces to approximately 60: this leads to a significance of approximately $1.0(2.6) \sigma$ with $100(1000) \mathrm{fb}^{-1}$ integrated luminosity. For $m_{H}=170 \mathrm{GeV}$, the raw event rate is approximately 80 to start with and, at the end, the count is only 212 . The total SM backgrounds is approximately 30, which leads to a significance approximately $0.4(1.23) \sigma$.

The LHeC will be operational for about ten years and expected to accumulate a total integrated luminosity of 1000 $\mathrm{fb}^{-1}$ of data. So, at the end of the run, we expect the SM Higgs boson will have $7.5 \sigma(3.8 \sigma)$ significance for Scenario Ib with $X=Z=44(28)$ with $Y=5(10)$. For Scenario IIa and Scenario Y, with $X=26$ and $Y=2$, the final significances are approximately $1 \sigma$. It seems that in all scenarios of the model, large $X$ are favorable. For heavy Higgs masses with $m_{H}=130 \mathrm{GeV}$, for Scenario Ib with $X=44$ and 30 with $Y=5$, the maximal significances are approximately 8.1 and $3.7 \sigma$ respectively. For $m_{H}=150 \mathrm{GeV}$, Scenario Ib with $X=44$ and $Y=5$, the final significance is $2.62 \sigma$. For $m_{H}=170$ $\mathrm{GeV}$, in the Scenario Ib with $X=30$ and $Y=5$, the final number of signal events is approximately 2. The estimated significances is $1.23 \sigma$. Thus, for high enough Higgs masses, one might invoke the aforementioned multivariate analyses to have larger significances.

\section{CONCLUSIONS}

After the discovery of a SM-like Higgs boson at LHC, one is well motivated to look for more such states, which necessarily appear in BSM scenarios. Among the experimental facilities where more Higgs bosons can be searched for, one should list an ep collider which may be built at CERN, known as the LHeC. In our analysis we have considered a 2HDM-III with a four-zero Yukawa texture in three configurations, wherein both the SM-like Higgs boson and the heavier version of it can be accessible at the foreseen LHeC energy. We assumed that both of these states are decaying via a flavor-violating mode $(\phi \rightarrow b \bar{s})$. After a parameter scan, we have selected a few model benchmarks where the products of cross sections and flavor-violating BRs are large enough to produce sufficient events in which to look for both signatures. We studied the three-jet and missing energy channel, $3 j+\mathbb{E}_{T}$, from the charged-current production of $\nu_{e} \phi q_{f}$, where $q_{f}$ is a forward jet with large rapidity and the other two jets come from the flavor-violating decay $\phi \rightarrow b \bar{s}$. We demanded one central jet to be $b$-tagged with the inclusion of the proper mis-tagging from light-flavor and gluon jets. We considered the most dominant SM backgrounds: charged-currents, $\nu t \bar{b}, \nu b \bar{b} j, \nu b 2 j$ and $\nu 3 j$, and photo-production, $e^{-} b \bar{b} j$ and $e^{-} t \bar{t}$. We performed a full hadron-level Monte Carlo simulation using CalcHEP as matrix element calculator, PYTHIA as parton shower/hadronization event generator and its PYCELL toy calorimeter in accordance with the LHeC detector parameters. We carefully implemented $b$-tagging, including mis-tagging of $c$-jets or light-flavor or gluon jets.

The signals under consideration do not have leptons, so we applied lepton vetos. The charged-current production has naturally missing energy due to the presence of neutrinos but no charged lepton. However, the photo-production processes have leptons in them but no direct missing energy (except the mis-measurements from jets and smearing), thus the missing energy selection together with the lepton veto suppressed the photo-production backgrounds to a very large extent.

The kinematics of the particular signals considered is very interesting from the fact that the Higgs boson is produced in the central rapidity region and its decay daughters, one $b$-jet and one light-flavor jet, are also central. We reconstructed the invariant mass of this two jets and selected events only for masses within a $15 \mathrm{GeV}$ window around the respective Higgs masses of the signal benchmarks. This selection reduces the SM backgrounds to a large extent and the invariant mass ensures the selection of flavor-violating decays. For massive Higgs bosons, although the signal events becomes low with the mass window selection, background suppression is more efficient.

12 Note that our selection cuts applied above are not optimized. An increase of the luminosity is an easy solution from a phenomenological perspective. However, adopting multivariate analysis techniques must also be a better discriminator of signal from backgrounds. 
As a next step of our selection, we identified the most energetic light-flavor forward jet (by seeing the rapidity profiles) and calculated the invariant mass with that jet together with the flavor-violating Higgs candidates jets. These three-jet invariant masses essentially give the overall energy scale of the hard scattering. Again, the more massive Higgs boson helps to suppress more SM backgrounds, in particular $\nu t \bar{b}$ and et $\bar{t}$, but the signal becomes smaller too.

At the end, the most important cut, we applied a central jet veto, i.e., we required one light-flavor central jet only. This suppresses SM backgrounds with large multiplicity, for example, $\nu t \bar{b}$ and $e t \bar{t}$.

After all the selections, with $100 \mathrm{fb}^{-1}$ of data, we found that the SM Higgs boson, $h$, would be detectable within the 2HDM-III in the scenario called in this work Ib with $X=Z=44$ or 30 with $Y=5$, with approximately $1-2 \sigma$. The heavier neutral Higgs boson, $H$, with masses $130 \mathrm{GeV}(150 \mathrm{GeV})$, would have $2(1) \sigma$ significances for large $X$ and only for Scenario Ib.

The LHeC will be operational for around ten years and so it is expected to accumulate a total integrated luminosity of $1000 \mathrm{fb}^{-1}$ of data. So, in all the cases mentioned above, the final significances will be enhanced. At the end of the run, the 2HDM-III Like-IIa (Like-Y) the SM Higgs will have $1 \sigma$. For $m_{H}=130 \mathrm{GeV}$, in the Scenario Ib with $X=44$ (30) and $Y=5$, the maximal significances are approximately $3.7(8.1) \sigma$. The maximal significances for $m_{H}=150$ $\mathrm{GeV}$ is $2.6 \sigma$ for $\mathrm{Ib}$ with $X=44$ and $Y=5$. For $m_{H}=170 \mathrm{GeV}$ the final number of signal events is approximately 2 , probably too little to be detected. However, it should be noted that we have adopted a simple cut-based method in this analysis. One would instead invoke more complex discriminators to enhance the significances within the designed luminosity, for example, multi-variate analyses.

To conclude, after the first few years of the LHeC running, by adopting more complex discriminator and/or multivariate analyses, we expect that both $h$ and $H$ signals will appear at the LHeC.

\section{ACKNOWLEDGMENTS}

This work has been supported by SNI-CONACYT (México), VIEP-BUAP and by PRODEP-SEP (México) under the grant: "Red Temática: Física del Higgs y del Sabor". RX acknowledges the scholarship from CONACYT (México). S.M. is supported in part through the NExT Institute. SPD acknowledges travel grant and all other supports from PRODEP-SEP (México): "Red Temática: Física del Higgs y del Sabor". SPD also acknowledges the visiting fellowship from FCFM and support from the project "New Physics with CMS". SPD is grateful to the post-doctoral fellowship and academic leave from Institute of Physics, Bhubaneswar, Odisha, India, while the project started.

[1] G. Aad et al. [ATLAS Collaboration], "Observation of a new particle in the search for the Standard Model Higgs boson with the ATLAS detector at the LHC," Phys. Lett. B 716, 1 (2012) arXiv:1207.7214 [hep-ex]].

[2] S. Chatrchyan et al. [CMS Collaboration], "Observation of a new boson at a mass of 125 GeV with the CMS experiment at the LHC", Phys. Lett. B 716, 30 (2012) arXiv:1207.7235 [hep-ex]].

[3] J. Adam et al. [MEG Collaboration], "New constraint on the existence of the $\mu^{+} \rightarrow e^{+} \gamma$ decay," Phys. Rev. Lett. 110, 201801 (2013) [arXiv:1303.0754] [hep-ex]].

[4] B. Aubert et al. [BaBar Collaboration], "Measurement of the Semileptonic Decays $B \rightarrow D \tau^{-} \bar{\nu}_{\tau}$ and $B \rightarrow D^{(*)} \tau^{-} \bar{\nu}_{\tau}, "$ Phys. Rev. D 79, 092002 (2009) arXiv:0902.2660 [hep-ex]]; J. P. Lees et al. [BaBar Collaboration], "Evidence for an excess of $\bar{B} \rightarrow D^{(*)} \tau^{-} \bar{\nu}_{\tau}$ decays," Phys. Rev. Lett. 109, 101802 (2012) arXiv:1205.5442 [hep-ex]]; J. P. Lees et al. [BaBar Collaboration], "Precision Measurement of the $B \rightarrow X_{s} \gamma$ Photon Energy Spectrum, Branching Fraction, and Direct CP Asymmetry $A_{C P}\left(B \rightarrow X_{s+d} \gamma\right)$," Phys. Rev. Lett. 109, 191801 (2012) arXiv:1207.2690 [hep-ex]]; J. P. Lees et al. [BaBar Collaboration], "Exclusive Measurements of $b \rightarrow s \gamma$ Transition Rate and Photon Energy Spectrum," Phys. Rev. D 86, 052012 (2012) arXiv:1207.2520 [hep-ex]].

[5] A. Matyja et al. [Belle Collaboration], "Observation of $B \rightarrow D^{*-} \tau^{+} \bar{\nu}_{\tau}$ decay at Belle," Phys. Rev. Lett. 99, 191807 (2007) arXiv:0706.4429 [hep-ex]]; K. Abe et al. [Belle Collaboration], "A Measurement of the branching fraction for the inclusive $B \rightarrow X_{s} \gamma$ decays with BELLE," Phys. Lett. B 511, 151 (2001) hep-ex/0103042; A. Limosani et al. [Belle Collaboration], "Measurement of Inclusive Radiative B-meson Decays with a Photon Energy Threshold of 1.7-GeV," Phys. Rev. Lett. 103, 241801 (2009) arXiv:0907.1384 [hep-ex]].

[6] G. C. Branco, P. M. Ferreira, L. Lavoura, M. N. Rebelo, M. Sher and J. P. Silva, "Theory and phenomenology of twoHiggs-doublet models," Phys. Rept. 516, 1 (2012) [arXiv:1106.0034 [hep-ph]].

[7] J. F. Gunion and H. E. Haber, "The CP conserving two Higgs doublet model: The Approach to the decoupling limit," Phys. Rev. D 67, 075019 (2003) hep-ph/0207010.

[8] A. Cordero-Cid, J. Hernandez-Sanchez, C. G. Honorato, S. Moretti, M. A. Perez and A. Rosado, "Impact of a four-zero Yukawa texture on $h \rightarrow \gamma \gamma$ and $\gamma Z$ in the framework of the Two Higgs Doublet Model Type III," JHEP 1407, 057 (2014) arXiv:1312.5614 [hep-ph]]. 
[9] O. Felix-Beltran, O. Flix-Beltrn, F. Gonzlez-Canales, J. Hernndez-Snchez, S. Moretti, R. Noriega-Papaqui and A. Rosado, "Analysis of the quark sector in the 2HDM with a four-zero Yukawa texture using the most recent data on the CKM matrix," Phys. Lett. B 742, 347 (2015) arXiv:1311.5210 [hep-ph]].

[10] J. Hernandez-Sanchez, S. Moretti, R. Noriega-Papaqui and A. Rosado, "Off-diagonal terms in Yukawa textures of the TypeIII 2-Higgs doublet model and light charged Higgs boson phenomenology," JHEP 1307, 044 (2013) arXiv:1212.6818.

[11] J. Hernandez-Sanchez, S. Moretti, R. Noriega-Papaqui and A. Rosado, "Update of the 2HDM-III with a four-zero texture in the Yukawa matrices and phenomenology of the charged Higgs Boson," PoS CHARGED 2012, 029 (2012) arXiv:1302.0083.

[12] J. Hernandez-Sanchez, C. G. Honorato, M. A. Perez and J. J. Toscano, "The $\gamma \gamma \rightarrow \phi_{i} \phi_{j}$ processes in the Type-III two-Higgs-doublet model," Phys. Rev. D 85, 015020 (2012) [arXiv:1108.4074 [hep-ph]].

[13] A. G. Akeroyd, S. Moretti and J. Hernandez-Sanchez, " $H^{ \pm} \rightarrow c b$ in models with two or more Higgs doublets," arXiv:1409.7596 [hep-ph].

[14] J. L. Diaz-Cruz, R. Noriega-Papaqui and A. Rosado, "Measuring the fermionic couplings of the Higgs boson at future colliders as a probe of a non-minimal flavor structure," Phys. Rev. D 71, 015014 (2005) hep-ph/0410391.

[15] J. L. Diaz-Cruz, R. Noriega-Papaqui and A. Rosado, "Mass matrix ansatz and lepton flavor violation in the THDM-III," Phys. Rev. D 69, 095002 (2004) hep-ph/0401194.

[16] J. L. Diaz-Cruz and J. J. Toscano, "Lepton flavor violating decays of Higgs bosons beyond the standard model," Phys. Rev. D 62, 116005 (2000) hep-ph/9910233.

[17] E. Arganda, A. M. Curiel, M. J. Herrero and D. Temes, "Lepton flavor violating Higgs boson decays from massive seesaw neutrinos," Phys. Rev. D 71, 035011 (2005) hep-ph/0407302; G. Blankenburg, J. Ellis and G. Isidori, "Flavour-Changing Decays of a 125 GeV Higgs-like Particle," Phys. Lett. B 712, 386 (2012) arXiv:1202.5704 [hep-ph]]; R. Harnik, J. Kopp and J. Zupan, "Flavor Violating Higgs Decays," JHEP 1303, 026 (2013) arXiv:1209.1397 [hep-ph]]; M. Arana-Catania, E. Arganda and M. J. Herrero, "Non-decoupling SUSY in LFV Higgs decays: a window to new physics at the LHC," JHEP 1309, 160 (2013) arXiv:1304.3371 [hep-ph]]; E. Arganda, M. J. Herrero, X. Marcano and C. Weiland, "Imprints of massive inverse seesaw model neutrinos in lepton flavor violating Higgs boson decays," Phys. Rev. D 91, no. 1, 015001 (2015) arXiv:1405.4300 [hep-ph]];

S. Bressler, A. Dery and A. Efrati, "Asymmetric lepton-flavor violating Higgs boson decays," Phys. Rev. D 90, no. 1, 015025 (2014) arXiv:1405.4545 [hep-ph]];

E. Arganda, M. J. Herrero, X. Marcano and C. Weiland, "Radiatively-induced LFV Higgs Decays from Massive ISS Neutrinos," arXiv:1410.5779 [hep-ph];

J. Heeck, M. Holthausen, W. Rodejohann and Y. Shimizu, "Higgs $\rightarrow \mu \tau$ in Abelian and Non-Abelian Flavor Symmetry Models," arXiv:1412.3671 [hep-ph]; A. Crivellin, G. D'Ambrosio and J. Heeck, "Explaining $h \rightarrow \mu^{ \pm} \tau^{\mp}, B \rightarrow K^{*} \mu^{+} \mu^{-}$ and $B \rightarrow K \mu^{+} \mu^{-} / B \rightarrow K e^{+} e^{-}$in a two-Higgs-doublet model with gauged $L_{\mu}-L_{\tau}$," Phys. Rev. Lett. 114, 151801 (2015) arXiv:1501.00993 [hep-ph]].

[18] D. Aristizabal Sierra and A. Vicente, "Explaining the CMS Higgs flavor violating decay excess," Phys. Rev. D 90, 115004 (2014) arXiv:1409.7690 [hep-ph]].

[19] J. Kopp and M. Nardecchia, "Flavor and CP violation in Higgs decays," JHEP 1410, 156 (2014) arXiv:1406.5303[hep-ph]].

[20] A. Greljo, J. F. Kamenik and J. Kopp, "Disentangling Flavor Violation in the Top-Higgs Sector at the LHC," JHEP 1407, 046 (2014) arXiv:1404.1278 [hep-ph]].

[21] A. Dery, A. Efrati, Y. Nir, Y. Soreq and V. Susi, "Model building for flavor changing Higgs couplings," arXiv:1408.1371 [hep-ph].

[22] A. Vicente, "Theory and phenomenology of lepton flavor violation," arXiv:1411.2372 [hep-ph].

[23] S. M. Boucenna, J. W. F. Valle and A. Vicente, "Predicting charged lepton flavor violation from gauge symmetry," arXiv:1502.07546 [hep-ph].

[24] M. Gomez-Bock and R. Noriega-Papaqui, "Flavor violating decays of the Higgs bosons in the THDM-III," J. Phys. G 32, 761 (2006) hep-ph/0509353.

[25] L. de Lima, C. S. Machado, R. D. Matheus and L. A. F. d. Prado, "Higgs Flavor Violation as a Signal to Discriminate Models," arXiv:1501.06923 [hep-ph].

[26] I. Dorsner, S. Fajfer, A. Greljo, J. F. Kamenik, N. Kosnik and I. Nisandzic, "New Physics Models Facing Lepton Flavor Violating Higgs Decays at the Percent Level," arXiv:1502.07784 [hep-ph].

[27] Y. Omura, E. Senaha and K. Tobe, "Lepton-flavor-violating Higgs decay $h \rightarrow \mu \tau$ and muon anomalous magnetic moment in a general two Higgs doublet model," arXiv:1502.07824 [hep-ph].

[28] G. Cvetic, S. S. Hwang and C. S. Kim, "One loop renormalization group equations of the general framework with two Higgs doublets," Int. J. Mod. Phys. A 14, 769 (1999) hep-ph/9706323; G. Cvetic, C. S. Kim and S. S. Hwang, "Higgs mediated flavor changing neutral currents in the general framework with two Higgs doublets: An RGE analysis," Phys. Rev. D 58, 116003 (1998) hep-ph/9806282.

[29] A. Fernandez, C. Pagliarone, F. Ramirez-Zavaleta and J. J. Toscano, "Higgs mediated Double Flavor Violating top decays in Effective Theories," J. Phys. G 37, 085007 (2010) arXiv:0911.4995 [hep-ph]].

[30] U. Cotti, L. Diaz-Cruz, C. Pagliarone and E. Vataga, "Search for the lepton flavor violating Higgs decay H - i tau mu at hadron colliders," eConf C 010630, P102 (2001) hep-ph/0111236.

[31] S. Bar-Shalom, A. Rajaraman, D. Whiteson and F. Yu, "Collider Signals of Maximal Flavor Violation: Same-Sign Leptons from Same-Sign Tops at the Tevatron," Phys. Rev. D 78, 033003 (2008) arXiv:0803.3795 [hep-ph]].

[32] F. Larios, R. Martinez and M. A. Perez, "Constraints on top quark FCNC from electroweak precision measurements," Phys. Rev. D 72, 057504 (2005) hep-ph/0412222. 
[33] K. A. Assamagan, A. Deandrea and P. A. Delsart, "Search for the lepton flavor violating decay A0 / H0 $\rightarrow$ tau+- mu-+ at hadron colliders," Phys. Rev. D 67, 035001 (2003) hep-ph/0207302].

[34] T. Han and D. Marfatia, " $h \rightarrow \mu \tau$ at hadron colliders," Phys. Rev. Lett. 86, 1442 (2001) hep-ph/0008141.

[35] N. Craig and S. Thomas, "Exclusive Signals of an Extended Higgs Sector," JHEP 1211, 083 (2012) arXiv:1207.4835 [hep-ph]].

[36] N. Craig, J. Galloway and S. Thomas, "Searching for Signs of the Second Higgs Doublet," arXiv:1305.2424 [hep-ph].

[37] B. Dumont, J. F. Gunion, Y. Jiang and S. Kraml, "Constraints on and future prospects for Two-Higgs-Doublet Models in light of the LHC Higgs signal," Phys. Rev. D 90, 035021 (2014) arXiv:1405.3584 [hep-ph]].

[38] E. Brownson, N. Craig, U. Heintz, G. Kukartsev, M. Narain, N. Parashar and J. Stupak, "Heavy Higgs Scalars at Future Hadron Colliders (A Snowmass Whitepaper)," arXiv:1308.6334 [hep-ex].

[39] B. Coleppa, F. Kling and S. Su, "Constraining Type II 2HDM in Light of LHC Higgs Searches," JHEP 1401, 161 (2014) arXiv:1305.0002 [hep-ph]].

[40] B. Holdom and M. Ratzlaff, "Distinctive Heavy Higgs Decays," arXiv:1412.1513 [hep-ph].

[41] [ATLAS Collaboration], "Search for Higgs bosons in Two-Higgs-Doublet models in the $H \rightarrow W W \rightarrow e \nu \mu \nu$ channel with the ATLAS detector," ATLAS-CONF-2013-027, ATLAS-COM-CONF-2013-005.

[42] V. Khachatryan et al. [CMS Collaboration], "Search for lepton-flavour-violating decays of the Higgs boson," arXiv:1502.07400 [hep-ex].

[43] A. R. Johansen and M. Sher, "The Electron/Muon Specific Two Higgs Doublet Model at e+ e- Colliders," arXiv:1502.00516 [hep-ph].

[44] https://lhec.web.cern.ch

[45] See for e.g., M.Klein, R.Yoshida: Collider Physics at HERA Prog.Part.Nucl.Phys. 61(2008)343-393.

[46] I. A. Sarmiento-Alvarado, A. O. Bouzas and F. Larios, "Analysis of the top-quark charged-current coupling at the LHeC," arXiv:1412.6679 [hep-ph].

[47] S. L. Glashow and S. Weinberg, Natural conservation laws for neutral currents. Phys. Rev. D 15, 1958 (1977); E. A. Paschos, "Diagonal Neutral Currents," Phys. Rev. D 15, 1966 (1977).

[48] J. Hernandez-Sanchez, L. Lopez-Lozano, R. Noriega-Papaqui and A. Rosado, "Couplings of quarks in the Partially Aligned 2HDM with a four-zero texture Yukawa matrix," Phys. Rev. D 85, 071301 (2012) arXiv:1106.5035 [hep-ph]].

[49] J. L. Diaz-Cruz, J. Hernandez-Sanchez, S. Moretti, R. Noriega-Papaqui and A. Rosado, Phys. Rev. D 79, 095025 (2009) arXiv:0902.4490 [hep-ph]].

[50] Y. Grossman, "Phenomenology of models with more than two Higgs doublets," Nucl. Phys. B 426, 355 (1994) hep-ph/9401311.

[51] A. G. Akeroyd, S. Moretti and J. Hernandez-Sanchez, "Light charged Higgs bosons decaying to charm and bottom quarks in models with two or more Higgs doublets," Phys. Rev. D 85, 115002 (2012) arXiv:1203.5769 [hep-ph]].

[52] M. Aoki, S. Kanemura, K. Tsumura and K. Yagyu, "Models of Yukawa interaction in the two Higgs doublet model, and their collider phenomenology," Phys. Rev. D 80, 015017 (2009) arXiv:0902.4665 [hep-ph]].

[53] I. F. Ginzburg and I. P. Ivanov, "Tree-level unitarity constraints in the most general 2HDM," Phys. Rev. D 72, 115010 (2005) hep-ph/0508020.

[54] A. Crivellin, A. Kokulu and C. Greub, "Flavor-phenomenology of two-Higgs-doublet models with generic Yukawa structure," Phys. Rev. D 87, no. 9, 094031 (2013) arXiv:1303.5877 [hep-ph]].

[55] O. Deschamps, S. Descotes-Genon, S. Monteil, V. Niess, S. T'Jampens and V. Tisserand, "The Two Higgs Doublet of Type II facing flavour physics data," Phys. Rev. D 82, 073012 (2010) arXiv:0907.5135 [hep-ph]].

[56] S. Kanemura, M. Kikuchi and K. Yagyu, "Fingerprinting the extended Higgs sector using one-loop corrected Higgs boson couplings and future precision measurements," arXiv:1502.07716 [hep-ph].

[57] A. Biswas and A. Lahiri, "Masses of physical scalars in two Higgs doublet models," arXiv:1412.6187 [hep-ph].

[58] D. Das, "New limits on $\tan \beta$ for 2HDMs with $\mathbf{Z}_{2}$ symmetry," arXiv:1501.02610 [hep-ph].

[59] A. Belyaev, N. D. Christensen and A. Pukhov, "CalcHEP 3.4 for collider physics within and beyond the Standard Model," Comput. Phys. Commun. 184, 1729 (2013) arXiv:1207.6082 [hep-ph]].

[60] O. Bruening and M. Klein, "The Large Hadron Electron Collider," Mod. Phys. Lett. A 28, no. 16, 1330011 (2013) arXiv:1305.2090 [physics.acc-ph]].

[61] J. L. Abelleira Fernandez et al. [LHeC Study Group Collaboration], "On the Relation of the LHeC and the LHC," arXiv:1211.5102 [hep-ex].

[62] J. L. Abelleira Fernandez et al. [LHeC Study Group Collaboration], "A Large Hadron Electron Collider at CERN: Report on the Physics and Design Concepts for Machine and Detector," J. Phys. G 39, 075001 (2012) arXiv:1206.2913 [physics.acc$\mathrm{ph}]$.

[63] Bruce Mellado for the LHeC Study Group, Journal of Physics: Conference Series 455 (2013) 012019.

[64] See for e.g. R B Appleby et al 2013 J.Phys.G: Nucl.Part.Phys.40 125004.

[65] J. Pumplin, D. R. Stump, J. Huston, H. L. Lai, P. M. Nadolsky and W. K. Tung, "New generation of parton distributions with uncertainties from global QCD analysis," JHEP 0207, 012 (2002) hep-ph/0201195.

[66] T. Sjostrand, S. Mrenna and P. Z. Skands, "PYTHIA 6.4 Physics and Manual," JHEP 0605, 026 (2006) hep-ph/0603175.

[67] J. R. Ellis, M. K. Gaillard and D. V. Nanopoulos, "A Phenomenological Profile of the Higgs Boson," Nucl. Phys. B 106, 292 (1976); J. M. LoSecco, "Higgs Boson Production in Neutrino Scattering," Phys. Rev. D 14, 1352 (1976); R. M. Godbole, "Trimuon Events Due to Neutrino and anti-neutrino Induced Production of Vector Mesons and Higgs Boson," Phys. Rev. D 18, 95 (1978); Z. Hioki, S. Midorikawa and H. Nishiura, "Higgs Boson Production in High-energy Lepton - Nucleon Scattering," Prog. Theor. Phys. 69, 1484 (1983); T. Han and H. C. Liu, "Production Of Charged And Neutral Higgs 
Bosons In High-energy Lepton Nucleon Interactions," Z. Phys. C 28, 295 (1985). J. Blumlein, G. J. van Oldenborgh and R. Ruckl, "QCD and QED corrections to Higgs boson production in charged current e p scattering," Nucl. Phys. B 395, 35 (1993) hep-ph/9209219.

[68] R. Kleiss and W. J. Stirling, "Tagging the Higgs," Phys. Lett. B 200, 193 (1988); V. D. Barger, T. Han and R. J. N. Phillips, "Improving the Heavy Higgs Boson Two Charged Lepton - Two Neutrino Signal," Phys. Rev. D 37, 2005 (1988); V. D. Barger, K. m. Cheung, T. Han and R. J. N. Phillips, "Strong $W^{+} W^{+}$scattering signals at $p p$ supercolliders," Phys. Rev. D 42, 3052 (1990).

[69] T. Han and B. Mellado, "Higgs Boson Searches and the H b anti-b Coupling at the LHeC," Phys. Rev. D 82, 016009 (2010) arXiv:0909.2460 [hep-ph]].

[70] W. Zhe, W. Shao-Ming, M. Wen-Gan, G. Lei and Z. Ren-You, "The Light MSSM neutral Higgs boson production associated with an electron and a jet at the LHeC," Phys. Rev. D 83, 055003 (2011) arXiv:1101.4987 [hep-ph]].

[71] S. P. Das and M. Drees, "CP-violating Supersymmetric Higgs at the Tevatron and LHC," Phys. Rev. D 83, 035003 (2011) arXiv:1010.3701 [hep-ph]]; S. P. Das and M. Drees, "CP-violating MSSM Higgs at Tevatron and LHC," J. Phys. Conf. Ser. 259, 012071 (2010) arXiv:1010.2129 [hep-ph]]; S. P. Das, A. Datta and M. Drees, "CP-violating Higgs at Tevatron," AIP Conf. Proc. 1078, 223 (2009) arXiv:0809.2209 [hep-ph]]. 